\title{
Characterizing three-dimensional features of vortex surfaces in the flow past a finite plate $\odot$
}

Cite as: Phys. Fluids 32, 011903 (2020); https://doi.org/10.1063/1.5134551

Submitted: 31 October 2019 . Accepted: 19 December 2019 . Published Online: 09 January 2020

Wenwen Tong (童文文) (D), Yue Yang (杨越) (D), and Shizhao Wang (王士召) (D)

COLLECTIONS

This paper was selected as Featured
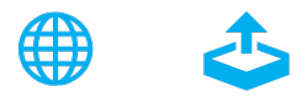

\section{ARTICLES YOU MAY BE INTERESTED IN}

Experimental study of hypersonic boundary layer transition on a permeable wall of a flared cone

Physics of Fluids 32, 011701 (2020); https://doi.org/10.1063/1.5139546

Hydrodynamics of median-fin interactions in fish-like locomotion: Effects of fin shape and movement

Physics of Fluids 32, 011902 (2020); https://doi.org/10.1063/1.5129274

Water exit dynamics of jumping archer fish: Integrating two-phase flow large-eddy simulation with experimental measurements

Physics of Fluids 32, 011904 (2020); https://doi.org/10.1063/1.5130886

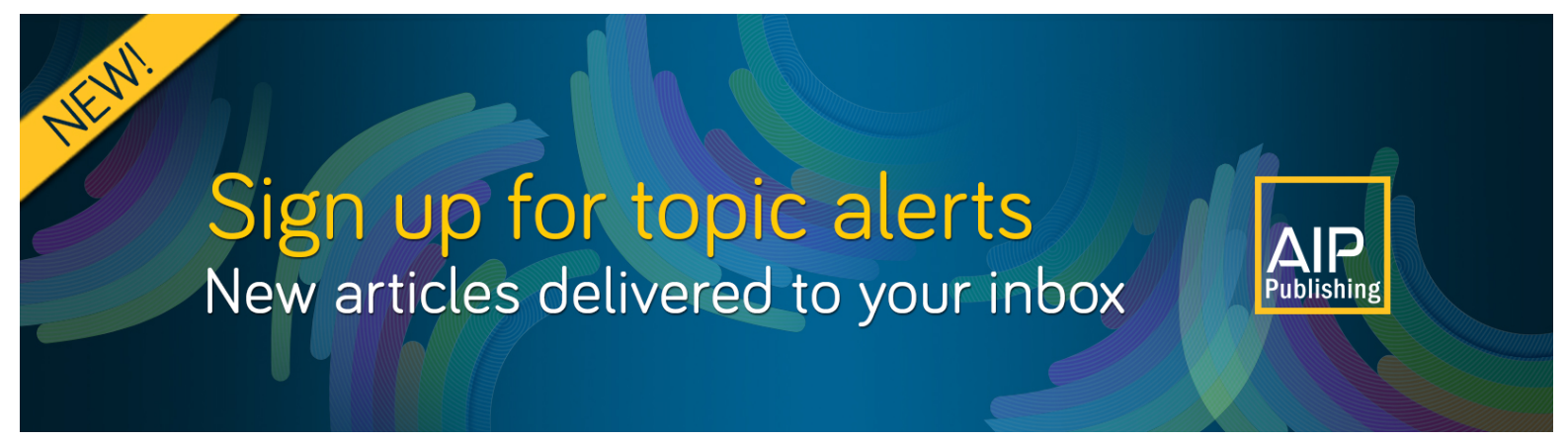




\title{
Characterizing three-dimensional features of vortex surfaces in the flow past a finite plate
}

\author{
Cite as: Phys. Fluids 32, 011903 (2020); doi: 10.1063/1.5134551 \\ Submitted: 31 October 2019 - Accepted: 19 December 2019 • \\ Published Online: 9 January 2020
}

Wenwen Tong (童文文), (D) Yue Yang (杨越), (2,2,a) (D) and Shizhao Wang (王士召) $)^{3}$

\begin{abstract}
AFFILIATIONS
'State Key Laboratory for Turbulence and Complex Systems, College of Engineering, Peking University, Beijing 100871, China ${ }^{2}$ CAPT and BIC-ESAT, Peking University, Beijing 100871, China

${ }^{3}$ LNM, Institute of Mechanics, Chinese Academy of Sciences, Beijing 100190, China and School of Engineering Sciences, University of Chinese Academy of Sciences, Beijing 100049, China
\end{abstract}

a)yyg@pku.edu.cn

\begin{abstract}
We extend the vortex-surface field (VSF), a Lagrangian-based structure identification method, to investigate vortex dynamics in flows past a plate simulated by the immersed boundary method. As an example, the VSF evolution characterizes the three-dimensional features of vortex surfaces in the flow past a finite plate at the Reynolds number of 300, aspect ratio of 2, and angle of attack of $30^{\circ}$. The VSF isosurface displays that near-plate vortex surfaces first roll up from plate edges and then evolve into hairpinlike structures near the leading edge and semiring structures near plate tips and in the wake. We quantitatively distinguish two types of vortical structures by the vanishing streamwise vorticity on VSF isosurfaces and refer them to as the leading edge vortex (LEV) and the tip vortex (TIV). Based on circulations through cross sections of vortex surfaces, we demonstrate that the lift generated from the LEV is suppressed by the finite growth of TIVs. In the wake region, we quantify the geometry of helical vortex lines in TIVs and the contribution of the helical vorticity component to the streamwise vortical impulse.
\end{abstract}

Published under license by AIP Publishing. https://doi.org/10.1063/1.5134551

\section{INTRODUCTION}

The flow past low-aspect-ratio wings with low and moderate Reynolds numbers is common in the application of micro air vehicles and unmanned aerial vehicles and in the natural animal locomotion such as birds and insects. ${ }^{1}$ The high lift and efficiency in unsteady flight relies on the generation of coherent vortical structures $^{2-4}$ with strong three-dimensional features, ${ }^{5-9}$ e.g., the leading edge vortex (LEV), tip vortex (TIV), and trailing edge vortex (TEV). The flow past a finite plate is a useful model for understanding the relationship between the vortical structures and forces and further improving flow control techniques. ${ }^{1}$

The interaction of the LEV and TIV plays an important role in unsteady flight of finite wings. Ellington et al. ${ }^{2}$ observed that the spanwise spiral flow toward wing tips can stabilize the LEV in the flow around wings of the hawkmoth using smoke visualization. The flow visualizations based on the particle image velocimetry (PIV) for flows past a rectangular plate ${ }^{16-18}$ indicated that the formation of the compressed LEV and bulgelike structures is caused by the inward spanwise flow of TIVs. Taira and Colonius ${ }^{19}$ carried out a systematic numerical study to analyze the effect of the angle of attack $\alpha$ and aspect ratio $R_{A}$ on the vortex topology for impulsively translated flat plates at low Reynolds numbers. They observed that the induced velocity from the TIV can keep the LEV attached to the plate with $R_{A}=1$, but the weaker performance of TIVs along the span cannot stabilize LEV for larger $R_{A}$. From analysis of vorticity dynamics, Lee et al. ${ }^{20}$ found that the LEV, TIV, and TEV form a ringlike structure for an impulsively started finite plate with $R_{A}=1$ $\sim 3$ at low Reynolds numbers. For the three-dimensional wake topology, double-loop vortices are generated for pitching-rolling plates ${ }^{21}$ or flapping plates ${ }^{22}$ and eventually form a bifurcating wake pattern. From the numerical simulation of the flow past a pitching lowaspect-ratio rectangular wing, $\mathrm{Visbal}^{23}$ found that the LEV evolves into an arch-type vortex and postulated that the upstream propagation of the arch vortex attributes to the self-induced velocity. Eldredge and Jones ${ }^{24}$ summarized that the underlying physics of the LEV formation and growth for a set of canonical wing motions is affected by the TIV. 
In flow wakes, the TIV forms helical structures and propagates downstream. The helical TIV can be generally quantified by swirl and axial velocities, and the geometry of helical vortex lines on a vortex tube can be characterized by the helix pitch and radius. ${ }^{25,26}$ In addition, the TIV can be simplified by axisymmetric vortex models, e.g., the Lamb-Oseen vortex ${ }^{27}$ and the Batchelor vortex, ${ }^{28}$ to examine the self-similarity of velocity profiles. Devenport et al. ${ }^{29}$ observed the self-similar wake spiral based on the velocity field in the evolution of TIVs for a rectangular wing. Spalart ${ }^{30}$ summarized the formation, motion, and persistence of airplane trailing vortices and pointed out that the helical shape of vortex lines induces jetlike axial flows at the wake. Okulov et al. ${ }^{31}$ studied the evolution of helical TIVs generated by a rotor and elucidated the helical self-similarity of the vortex cores and helical vortex development using the axial vorticity and swirl velocity.

The development of the LEV and TIV is related to lift and drag for finite wings. Wu et al. summarized fundamental theories of aerodynamic forces in viscous flows from the structure level, such as the vortex-force theory and the impulse theory based on velocity and vorticity fields. ${ }^{32}$ The Kutta-Joukowski theorem can estimate the circulatory lift force using the circulation of the LEV on the spanwise cross section. Wang et al. ${ }^{33}$ developed a wake-sectional Kutta-Joukowski model to predict the lift from wake velocity data. For a given vorticity field, the vorticitymoment theory can be used to obtain the force acting on a body using the vortical impulse in the entire flow field. ${ }^{34}$ Recently, the minimum-domain impulse theory was developed to minimize the integral domain for the vortical impulse, ${ }^{35}$ but the objective choice of the integral domain for the LEV and TIV is still an open issue. Moreover, the scaling relation between the propulsive performance and Strouhal numbers is established to elucidate the flow physics behind the thrust generation from the topology of shedding vortices. $^{36,37}$

In the previous studies, the LEV and TIV are usually not accurately defined and their boundary is ambiguous, partially owing to the issue of the vortex identification. ${ }^{38,39}$ Therefore, the quantitative study on the evolution of the LEV and TIV and the characterization of their interaction is still lacking. Moreover, although the helical vortex lines of the TIV have been observed, their implication on lift and drag forces has not been clearly quantified. Thus, we need an effective tool to characterize the helical part of the TIV in the wake and further elucidate its contribution to the aerodynamic forces.

The Eulerian vortex identification methods based on the local velocity gradient, such as $Q-, \Delta$-, and $\lambda_{2}$-criteria, ${ }^{40-42}$ are able to identify instantaneous vortex cores, but, in principle, they cannot ensure the time coherence identified structures. Furthermore, the ambiguous boundary of the LEV and TIV results in the difficulty for clarifying their interaction and contributions to lift and drag forces.

In order to characterize the continuous evolution of vortical structures, Yang and Pullin ${ }^{43,44}$ developed the vortex-surface field (VSF), whose isosurface is a vortex surface consisting of vortex lines. This method establishes a framework for the quantitative analysis of Lagrangian-based vortex dynamics. As a flow diagnostic tool, the VSF can be employed to the database of the numerical simulation and has been applied to wall-bounded flows for elucidating transition mechanisms from the deformation and reconnection of vortex surfaces. ${ }^{45-47}$ Other Lagrangian-based methods, e.g., the Lagrangian finite-time Lyapunov exponent, were employed to illuminate the formation and shedding of coherent structures in the flow past a flat plate, ${ }^{48,49}$ though they are not directly related to the vorticity and vortex forces. The Lagrangian-based methods appear to be more natural to study evolving flow structures than the Eulerian identification methods.

In this study, we extend the VSF method to the flow past a finite plate. First, we develop the numerical method for constructing VSFs in flows with an immersed boundary. ${ }^{50,51}$ Then, we characterize three-dimensional features of vortex surfaces in the flow evolution: (1) distinguish the LEV and TIV by introducing a mathematical definition of their boundary, (2) quantify the competition between the LEV and TIV and the streamwise development of the LEV, and (3) quantify the helical degree of TIV tubes in the wakes and elucidate the effect of helical vortex lines on drag and lift.

The outline of this paper is as follows: In Sec. II, we extend the two-time method ${ }^{44}$ to calculate VSFs in complex flows computed by the immersed boundary method. In Sec. III, we describe the evolution of VSFs based on the morphology of vortex surfaces and lines. Then, we quantify the competition between the LEV and TIV in Sec. IV and the contribution of helical vortex lines in TIVs to vortical impulses in Sec. V. Some conclusions are drawn in Sec. VI.

\section{SIMULATION OVERVIEW}

\section{A. Immersed boundary method}

The constant-density, incompressible flow past a finite plate is governed by Navier-Stokes equations

$$
\begin{aligned}
\frac{\partial \boldsymbol{u}}{\partial t}+\boldsymbol{u} \cdot \nabla \boldsymbol{u} & =-\nabla p+\frac{1}{R e} \nabla^{2} \boldsymbol{u}+\boldsymbol{f}, \\
\nabla \cdot \boldsymbol{u} & =0
\end{aligned}
$$

where $\boldsymbol{u}, p$, and $\boldsymbol{f}$ denote the nondimensional velocity, pressure, and Eulerian force, respectively, and $R e=U_{\infty} c / v$ denotes the Reynolds number with the reference velocity $U_{\infty}$ of incoming flow, the reference chord length $c$, and the kinematic viscosity $v$.

The immersed boundary method with the discrete stream function $^{51}$ is used to solve Eqs. (1) and (2). The immersed boundary is represented by Lagrangian markers. The regularized delta function $\delta_{h}{ }^{50}$ is employed to interpolate and spread $\boldsymbol{f}$ between Eulerian points and Lagrangian points, whose coordinates are denoted by $\boldsymbol{x}$ and $\boldsymbol{X}$, respectively. The Lagrangian points are uniformly distributed on the immersed boundary, and the distance between two neighboring Lagrangian points is close to the spacing of the nearby Eulerian mesh.

The Eulerian force in Eq. (1) is calculated as

$$
\boldsymbol{f}(\boldsymbol{x})=\int_{S} \boldsymbol{F}(\boldsymbol{X}) \delta_{h}(\boldsymbol{x}-\boldsymbol{X}) d \boldsymbol{X},
$$

where $\boldsymbol{F}$ denotes the Lagrangian force at $\boldsymbol{X}$ and $S$ is the domain of the immersed boundary. The Lagrangian force $\boldsymbol{F}$ is exerted on the immersed boundary to satisfy the no-slip condition, and the velocity on the immersed boundary satisfies 


$$
\int_{\Omega} \boldsymbol{u}(\boldsymbol{x}) \delta_{h}(\boldsymbol{x}-\boldsymbol{X}) d \boldsymbol{x}=\boldsymbol{U}_{b}(\boldsymbol{X}),
$$

where $\Omega$ denotes the entire fluid domain and $\boldsymbol{U}_{b}$ denotes a specified velocity at Lagrangian points. In this way, the information of the force and velocity on Eulerian and Lagrangian points is exchanged through Eqs. (3) and (4).

In the present simulation of the three-dimensional flow past a stationary finite flat plate, we set parameters $R_{A}=2, \alpha=30^{\circ}$, and $R e=300$. The uniform inflow velocity is specified at the inlet, and the fixed pressure condition is prescribed at the outlet. The slip-wall condition is used at the other four boundaries of $\Omega$. The flow is uniform with $\boldsymbol{u}=\left(U_{\infty}, 0,0\right)$ in $\Omega$ at $t=0$ before the plate instantaneously appears at $t=0^{+}$. Our in-house numerical code for simulating the flow with an immersed boundary has been validated, including the accuracy of the near-wall vorticity ${ }^{52}$ and the flow past an impulsively started plate.

As sketched in Fig. 1, two reference systems with the origin $O$ at the plate center are adopted in following analysis. In the natural reference system $O-x y z$, the $x$-axis is aligned with the incoming flow, the $y$-axis and the $z$-axis are aligned with the wing-span and vertical directions, respectively. In the plate-oriented reference system $O-x_{1} x_{2} x_{3}, x_{1}, x_{2}$, and $x_{3}$ are tangent, spanwise, and normal directions to the plate, respectively. All the spatial coordinates in the two reference systems are nondimensionalized by the chord length $c$ in the following study.

As sketched in Fig. 2, the simulation is performed in a rectangular domain of $\Omega \in[-4,6.4] \times[-5,5] \times[-5,5]$. A locally refined mesh with the minimum spacing $\Delta x=0.02$ and the maximum spacing $\Delta x$ $=0.08$ is applied around the plate to achieve the high spatial resolution near the immersed boundary. The total number of grid points is $6.51 \times 10^{6}$. Our convergence test (not shown) for the immersed boundary method indicates that both drag and lift coefficients are converged on the present mesh, and $\Omega$ is large enough to avoid the boundary effect.

\section{B. VSF method}

The VSF $\phi_{v}$ is defined to satisfy the constraint

$$
\omega \cdot \nabla \phi_{v}=0
$$

so that every isosurface of $\phi_{v}$ is a vortex surface consisting of vortex lines. ${ }^{43}$ The calculation of VSFs is implemented as a postprocessing

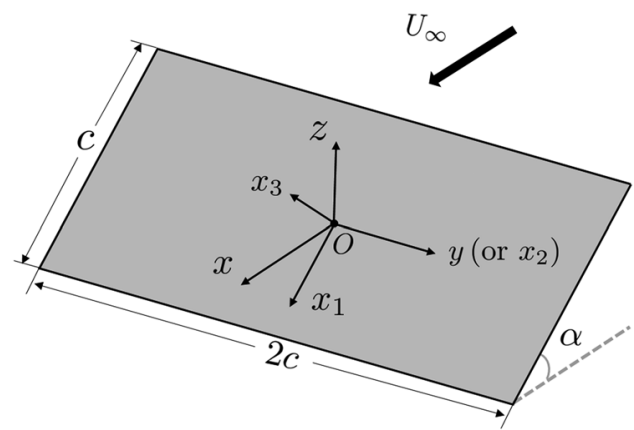

FIG. 1. The schematic diagram of a flow past a finite plate with two coordinate systems $0-x y z$ and $0-x_{1} x_{2} x_{3}$.

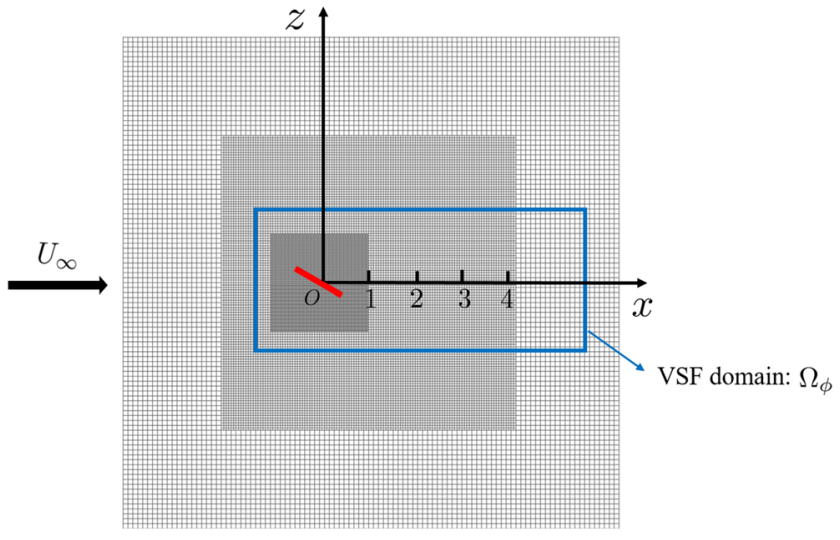

Flow-field domain: $\Omega$

FIG. 2. The sketch of flow-field and VSF domains on the $x-z$ plane at $y=0$. The red line represents the plate.

step based on a time series of velocity-vorticity fields obtained by solving Eqs. (1) and (2).

The two-time method ${ }^{44}$ is used for calculating a Lagrangianlike temporal evolution of VSFs. For each physical time step, this method involves prediction and correction substeps. In the prediction substep, the temporary VSF solution $\phi_{v}^{*}$ is advanced in the physical time as

$$
\frac{\partial \phi_{v}^{*}(\boldsymbol{x}, t)}{\partial t}+\boldsymbol{u}(\boldsymbol{x}, t) \cdot \nabla \phi_{v}^{*}(\boldsymbol{x}, t)=0, \quad t \geq 0
$$

where $\boldsymbol{u}$ is the Eulerian velocity, and $\phi_{v}^{*}$ can slightly deviate from an accurate VSF. In the correction substep, the temporary VSF solution $\phi_{v}^{*}$ is transported along the frozen vorticity in pseudotime as

$$
\frac{\partial \phi_{v}(\boldsymbol{x}, t ; \tau)}{\partial \tau}+\boldsymbol{\omega}(\boldsymbol{x}, t) \cdot \nabla \phi_{v}(\boldsymbol{x}, t ; \tau)=0, \quad 0 \leq \tau \leq T_{\tau},
$$

with the initial condition

$$
\phi_{v}(x, t ; \tau=0)=\phi_{v}^{*}(x, t) .
$$

Finally, $\phi_{v}$ is updated by $\phi_{v}\left(\boldsymbol{x}, t ; \tau=T_{\tau}\right)$ with pseudotime evolution, where $T_{\tau}$ is the maximum value of pseudotime to ensure the convergence of $\phi_{v}$ in Eq. (7), and it is typically less than 100 times of the time step $\Delta t$ for Eq. (6).

As sketched in Fig. 2, the VSF calculation is carried out within a subdomain $\Omega_{\phi}$ around the immersed body and the near-wake region with strong vorticity. A high-resolution, uniform mesh is used for VSF calculation, ensuring the smoothness of VSF solutions. In $\Omega_{\phi}$, velocity and vorticity fields are interpolated from the unstructured mesh in $\Omega$ by the quadratic Shepard method ${ }^{54}$ based on scattered data interpolation. For balancing the VSF deviation and computational cost, we choose $\Omega_{\phi} \in[-1,6] \times[-1.5,1.5] \times[-2,1]$ and $\Delta x$ $=0.02$. The mesh spacing in $\Omega_{\phi}$ is equal to the minimum one for the unstructured mesh in $\Omega$. 
(a)

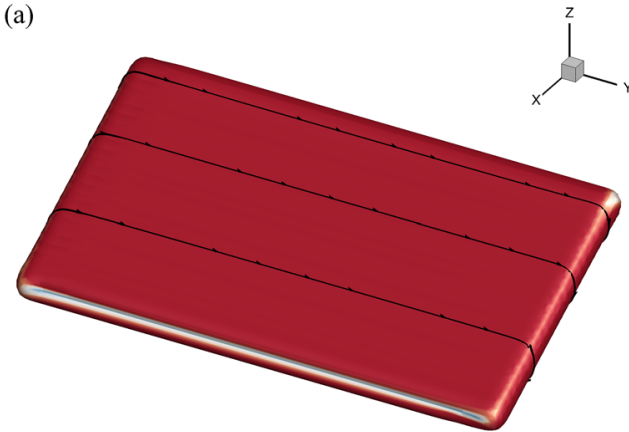

(b)

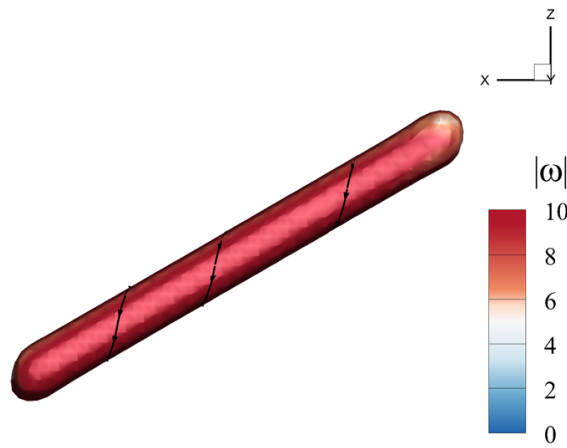

FIG. 3. Isosurface of $\phi_{v 0}=0.5$ colorcoded by the vorticity magnitude at $t_{0}$ $=0.02$. (a) Perspective view and (b) side view.

\section{Extension of VSF construction to the immersed boundary method}

We extend the VSF, which was only applied to flows with simple boundary conditions, ${ }^{46,55}$ to the immersed boundary condition, which can have complex geometries. Since the normal vorticity on the plate is vanishing owing to the no-slip condition, the plate is a vortex surface with all the vortex lines attached on its surface. Thus, we enforce constant $\phi_{v}=\phi_{v}^{B}$ on the plate, i.e., the Dirichlet boundary condition for VSF.

Similar to the treatment of the temperature at the immersed boundary in heat transfer problems, ${ }^{56,57}$ we add a source term to Eq. (6) to satisfy $\phi_{v}=\phi_{v}^{B}$ at the immersed boundary as

$$
\frac{\partial \phi_{v}^{*}(\boldsymbol{x}, t)}{\partial t}+\boldsymbol{u}(\boldsymbol{x}, t) \cdot \nabla \phi_{v}^{*}(\boldsymbol{x}, t)=q(\boldsymbol{x}),
$$

where $q(\boldsymbol{x})$ is the external VSF source term on Eulerian grid points. Since the vorticity is generated from the plate surface and then convected to wake flows, the immersed boundary can be considered as a source of the VSF. The numerical implementation of solving Eq. (9) and determining the value of $q(\boldsymbol{x})$ is described in Appendix $\mathrm{A}$ in detail. Similar to Eq. (9), we add a source term $q_{\tau}(\boldsymbol{x})$ to Eq. (7) as

$$
\frac{\partial \phi_{v}(\boldsymbol{x}, t ; \tau)}{\partial \tau}+\boldsymbol{\omega}(\boldsymbol{x}, t) \cdot \nabla \phi_{v}(\boldsymbol{x}, t ; \tau)=q_{\tau}(\boldsymbol{x}) .
$$

The interpolation and spreading operation between the immersed boundary and the nearby Eulerian flow field are based on the regularized delta function as

$$
\begin{aligned}
Q(\boldsymbol{X}) & =\int_{\Omega_{\phi}} q(\boldsymbol{x}) \delta_{h}(\boldsymbol{x}-\boldsymbol{X}) d \boldsymbol{x}, \\
q(\boldsymbol{x}) & =\int_{S} Q(\boldsymbol{X}) \delta_{h}(\boldsymbol{x}-\boldsymbol{X}) d \boldsymbol{X},
\end{aligned}
$$

where $Q(X)$ is the VSF source defined on the Lagrangian points $\boldsymbol{X}$ at the immersed boundary.

For solving Eqs. (9) and (10), the marching of $t$ and $\tau$ is approximated by the third-order total-variation-diminishing Runge-Kutta method, and the time and pseudotime steps satisfy the CFL conditions based on $\boldsymbol{u}$ and $\boldsymbol{\omega}$, respectively. The convection term is approximated by the fifth-order weighted essentially nonoscillatory scheme, where the numerical diffusion serves as a numerical dissipative regularization for smoothing nearly singular structures in VSF solutions.
An initial VSF $\phi_{v 0}$ needs to be specified for solving Eq. (9), and in general, exact $\phi_{v 0}$ satisfying Eq. (5) does not exist for an arbitrary given vorticity field. ${ }^{43}$ The present flow has $\boldsymbol{\omega}=0$ at $t=0$, and then, $\boldsymbol{\omega}$ is immediately generated on the plate at $t>0$. Since the vorticity dominates in the spanwise direction and diffuses along the wallnormal direction, it satisfies $\boldsymbol{\omega} \cdot \nabla|\boldsymbol{\omega}| \approx 0$. From the VSF definition [Eq. (5)], $|\omega|$ is close to an exact VSF at very early times, so we use $|\omega|$ at $t_{0}=0.02$ for constructing $\phi_{v 0}$. The pseudoevolution equation (10) is applied with the pseudoinitial condition

$$
\phi_{v 0}\left(\boldsymbol{x}, t_{0} ; \tau=0\right)=\sqrt{\frac{|\boldsymbol{\omega}(\boldsymbol{x})|}{|\boldsymbol{\omega}(\boldsymbol{x})|_{\max }}},
$$

where $|\boldsymbol{\omega}(\boldsymbol{x})|_{\max }$ is the maximum vorticity magnitude in $\Omega_{\phi}$. Then, the VSF at $t_{0}$ is obtained as

$$
\phi_{v 0}=\phi_{v 0}\left(\boldsymbol{x}, t_{0} ; \tau=T_{\tau}\right),
$$

and its isosurfaces are displayed in Fig. 3. We observe that all the ringlike vortex lines are almost along the spanwise direction, and the vortex surfaces with large $|\omega|$ warp up the plate.

The deviation of the numerical VSF solution from the exact VSF is defined by the cosine of angle between the VSF gradient and vorticity $^{43}$ as

$$
\lambda_{\omega} \equiv \frac{\boldsymbol{\omega} \cdot \nabla \phi_{v}}{|\boldsymbol{\omega}|\left|\nabla \phi_{v}\right|} .
$$

From the constructed initial VSF $\phi_{v 0}$, the VSF is evolved by the two-time method with a source term. Our convergence test for VSF solutions (not shown) demonstrated that $\left\langle\left|\lambda_{\omega}\right|\right\rangle$ is converged at large pseudotimes, and the converged value decreases with the VSF mesh resolution, consistent with the theoretical analysis. ${ }^{44}$ For the present VSF mesh with $\Delta x=0.02,\left\langle\left|\lambda_{\omega}\right|\right\rangle$ is controlled around $2 \% \sim 3 \%$, which is accurate enough for further characterization of vortex surfaces.

\section{TEMPORAL EVOLUTION OF THE VSF}

\section{A. Morphology of VSF isosurfaces}

The temporal evolution of the VSF isosurface with $\phi_{v}=0.5$ is shown in Fig. 4. At the early time $t=t_{0}$, the vortex surface remains flat and spanwise vortex lines are gradually curved and stretched along the streamwise direction in Fig. 4(a). Then, near-wall vortex 
(a)
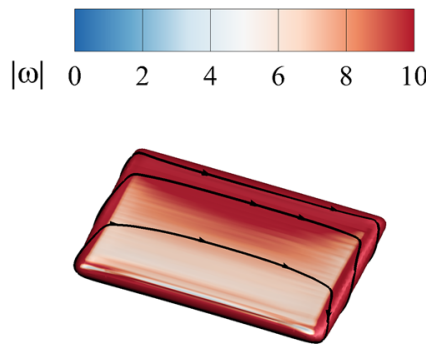

(c)

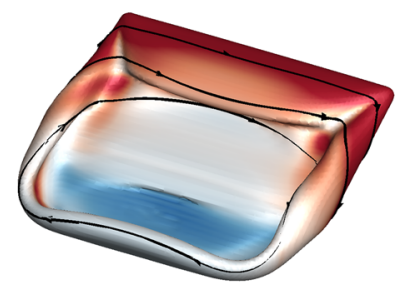

(e)

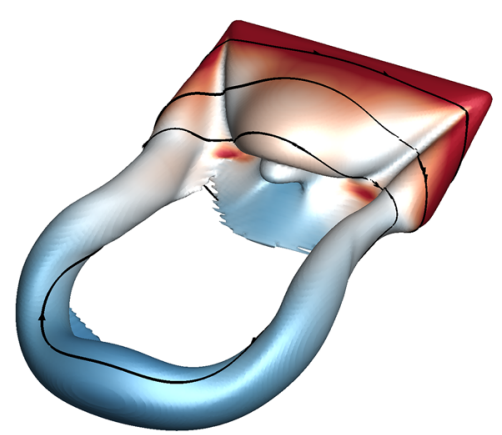

(g)

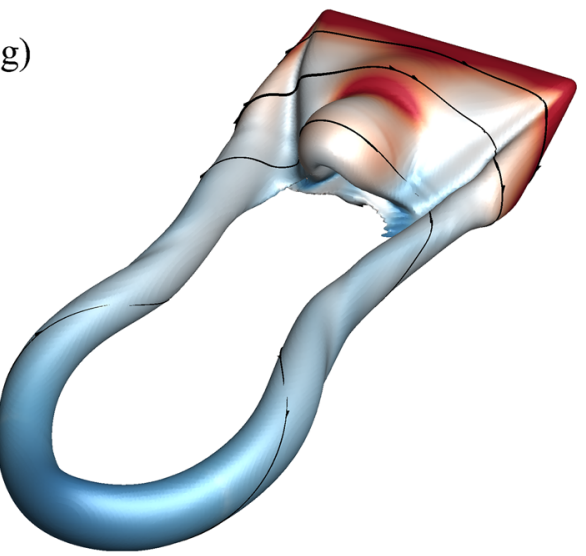

(b)<smiles></smiles>

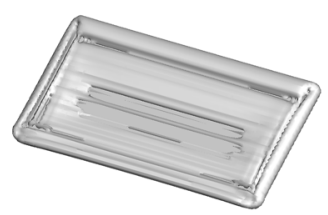

(d)

(f)
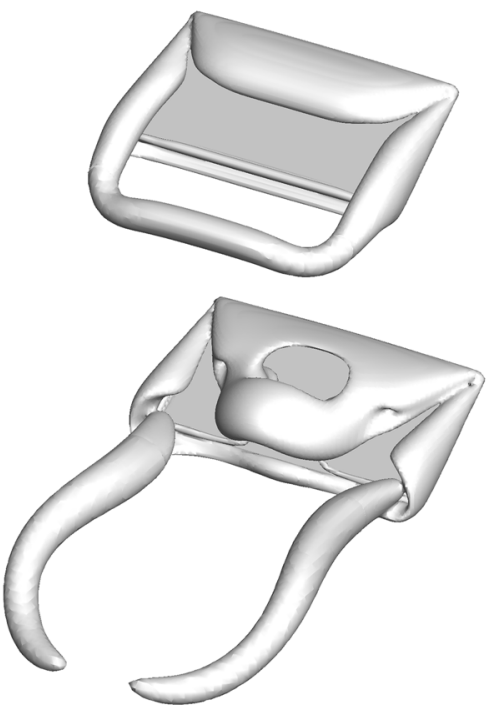

(h)

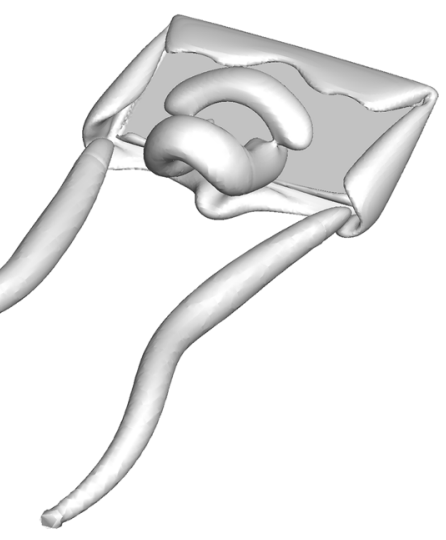

FIG. 4. Temporal evolution of isosurfaces of $\phi_{v}=0.5$ and $Q=3$. (a) $\phi_{v}, t=0.1$; (b) $Q, t=0.1$; (c) $\phi_{v}, t=1$; (d) $Q, t=1$; (e) $\phi_{v}, t=3$; (f) $Q, t=3$; (g) $\phi_{v}, t=5$; and (h) $Q, t=5$. The VSF isosurface is color-coded by the vorticity magnitude with some vortex lines integrated on the surface. surfaces roll up at plate edges when the incoming flow moves around the plate in Fig. 4(c). The vortex surface at the leading edge is lifted from the plate and forms a bulgelike structure. At the meantime, the vortex surface at the wake is stretched along the incoming flow direction and forms a semiring structure in Fig. 4(e). At the later time $t=5$, the hairpinlike structure is formed in the middle of the plate in Fig. 4(g). The semiring structure is significantly stretched and twisted in the wake region, with attached helical vortex lines interweaving with each other.

Compared with Eulerian vortex-identification methods, the VSF rooted in the Helmholtz theorem ${ }^{44}$ has stronger time coherence to display the temporal evolution of a particular vortex surface. In the comparison of isosurfaces of $\phi_{v}=0.5$ and the $Q$-criterion $Q=3$ in Fig. 4, we observe that the VSF displays complete vortex surfaces 
with perfectly attached vortex lines, whereas the isosurface of $Q$ only identifies vortex cores and loses some parts of vortex surfaces, which can be important in the further quantification of vortex dynamics and interaction.

In particular, we observe that a pair of concave structures with relatively weak $|\omega|$ gradually forms and extends from corners of the leading edge to the center of the plate. These structures can serve as a boundary distinguishing vortical structures developed from the leading edge and the tip region, which is elaborated in Sec. IV. Near the wake, the VSF isosurface identifies twisted vortex tubes in Figs. 4(g) and 4(h), and we find that the helical vortex lines on VSF isosurfaces play an important role in the propulsion efficiency, which is discussed in Sec. V.

Based on the morphology of vortex surfaces and lines in Fig. 4, we divide the VSF evolution into three stages: (1) streamwise stretching of vortex lines on the plate, (2) rolling up of near-wall vortex surfaces, and (3) formation of the hairpinlike vortex and helical vortex tubes. The signature structures and transition processes during these stages will be quantified by the VSF and attached vortex lines.

\section{B. Streamwise stretching of vortex lines}

At the initial time $t=t_{0}$, vortex surfaces are flat with vortex lines along the spanwise direction, except in the tip regions. The evolution of vortex surfaces/lines in the three-dimensional flow past a plate at early times can be approximated by the combination of twodimensional potential flows ${ }^{58}$ on $x_{1}-x_{3}$ and $x_{2}-x_{3}$ planes, except for the region near edges.

In Fig. 5, we decompose the incoming flow velocity $U_{\infty}=U_{\|}+$ $U_{\perp}$ into velocities tangent and normal to the plate and approximate the velocity close to edges as

$$
u_{1}\left(x_{1}, x_{3}= \pm 0\right)=U_{\infty} \cos \alpha \mp \frac{x_{1} U_{\infty} \sin \alpha}{\sqrt{(c / 2)^{2}-x_{1}^{2}}}
$$

and

$$
u_{2}\left(x_{2}, x_{3}= \pm 0\right)=\mp \frac{x_{2} U_{\infty} \sin \alpha}{\sqrt{c^{2}-x_{2}^{2}}} .
$$

Thus, the fluid flows inward to the suction side along edges of the plate, and the spanwise velocity decreases from the near-tip region to the middle of the plate. For the streamwise velocity, the velocity gradient along $x_{1}$ in Fig. 5(a) has

$$
\frac{\partial u_{1}}{\partial x_{1}}>0, x_{3}<0 \text { and } \frac{\partial u_{1}}{\partial x_{1}}<0, x_{3}>0
$$

At $t=t_{0}$, the vorticity is dominated by $\omega_{2}$ owing to the velocity gradient $\partial u_{1} / \partial x_{3}$ in the middle of the plate, as shown in Fig. 3(a). Near the near-tip region, e.g., at $x_{2}>1$, both $u_{3}$ and $u_{1}$ increase along the $x_{2}$-direction, so vorticity components can be approximated as

$$
\omega_{1} \simeq \frac{\partial u_{3}}{\partial x_{2}}>0 \text { and } \omega_{3} \simeq-\frac{\partial u_{1}}{\partial x_{2}}<0 .
$$

Thus, vortex lines on the tip are tilting from the $x_{1}$-direction in Fig. 3(b).

At this stage, the velocity distribution in Eq. (17) on the plate surface leads to the streamwise stretching of vortex lines shown in Fig. 3. In Fig. 4(a), we observe that the spanwise vortex lines are stretched toward upstream in the central region and near the trailing edge, and the streamwise stretching of tilting vortex lines at the tips is intensified.

\section{Rolling up of near-wall vortex surfaces}

After the strong vorticity is generated from the plate, the initial wall-parallel vortex surfaces gradually roll up and lift, forming threedimensional vortical structures near plate edges. The rolling up of an ideal vortex sheet and the finite growth of vortex-core radius in a two-dimensional plane is characterized in the Kaden problem. ${ }^{59}$ In three-dimensional flows, the plate is wrapped up by VSF isosurfaces with $0 \leq \phi_{v} \leq 1$, so the rolling up of vortex sheets is naturally characterized by the evolution of VSF isosurfaces.

Figure 6(a) shows the rolling up of VSF isosurfaces around the tip with intense swirl motion on the $y-z$ plane at $x=0.3$. We define the maximum vertical displacement of an isosurface of $\phi_{v}$ as

$$
\Delta z\left(\phi_{v}\right)=\max z\left(x, \phi_{v}\right)-z_{0}(x)
$$

where $z_{0}(x)$ denotes the $z$-coordinate of the plate on the $y$ - $z$ plane. In Fig. 6(b), all the profiles of $\Delta z\left(\phi_{v}\right)$ for various $\phi_{v}$ increase with time and reach a steady state at later times, indicating the finite growth of vortex rolls near the tip, and the vortex surface close to the wall with smaller $\phi_{v}$ is lifted higher with larger $\Delta z\left(\phi_{v}\right)$. The finite vortex size implies that the intensity of vortical structures created from the tip is limited, whose effect on the development of vortices generated from the leading edge is explained in Sec. IV.

After the rolling up of vortex surfaces at edges, the initial wallparallel vortex surfaces evolve into a three-dimensional dustpanlike structure at $t=1$. In Fig. 4(c), the complex vortical structure, except at plate corners, can be considered as a combination of vortical structures in two cross sections in potential flows in Fig. 5. The three-dimensional effect of the finite plate is manifested as the (a)

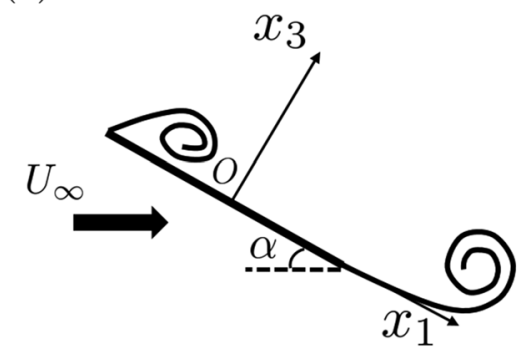

(b)

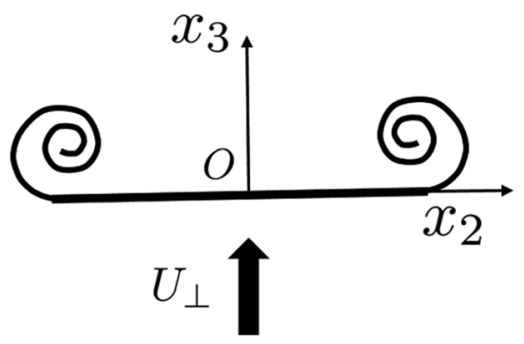

FIG. 5. Two-dimensional flow patterns on slices in the flow past a finite plate at early times. (a) $x_{1}-x_{3}$ plane and (b) $x_{2}-x_{3}$ plane. 
(a)

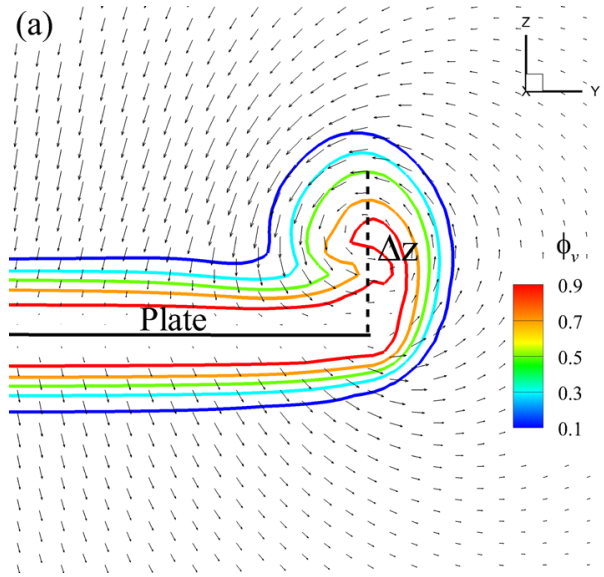

(b)

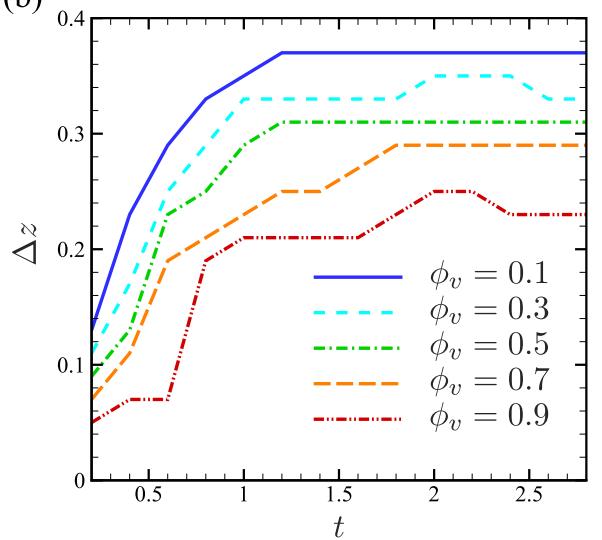

FIG. 6. Rolling up of VSF isosurfaces around $y=1$ on the $y-z$ plane at $x=0.3$. (a) Contour lines of $\phi_{v}$ at $t=1.0$ and (b) the temporal evolution of the maximum vertical displacement. bulgelike structure at the leading edge and the streamwise stretching of tilting vortex lines at the tips, which, respectively, evolve into the hairpinlike structure and helical vortex tubes in the next stage.

\section{Formation of the hairpinlike structure and helical vortex tubes}

After the rolling up of near-wall vortex surfaces, signature vortical structures gradually form near plate edges. Near the leading edge, the structures created from the leading edge and tips compete with each other. Near the wake region, the structures generated from the trailing edge and tips shed off and propagate downstream.

The three-dimensional effect of the finite plate becomes notable after the rolling-up of vortex tubes. In Fig. 6(a), the velocity distribution in the tip region on the $y-z$ plane indicates that the vortex tubes rolled-up from the tips can inhibit the vertical growth of the vortical structures created from the leading edge. ${ }^{20}$ The suppression of the vortex tubes at the tips on the central bulgelike structures results in a pair of slanting grooves extending from the corners at the leading edge in Fig. 4(c), and typical vortex lines on the VSF isosurface are reversed in the streamwise direction in Fig. 7. Thus, the isosurface of $\omega_{x}=0$ can distinguish the entire vortex surface into two parts. The

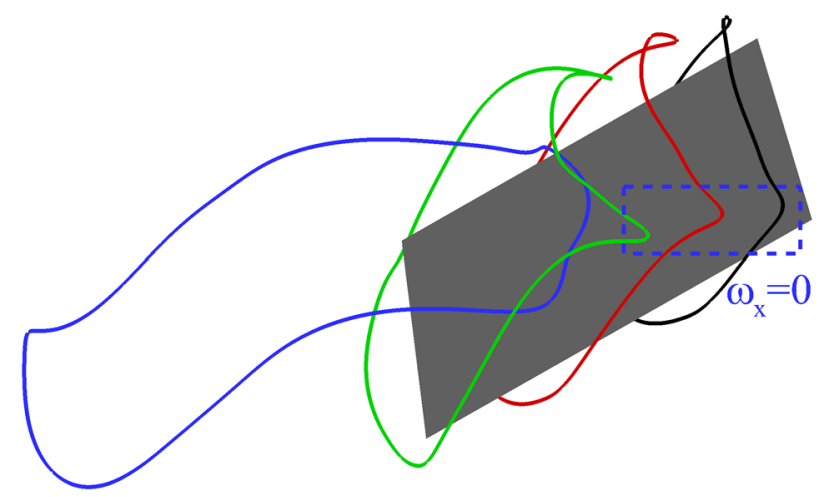

FIG. 7. The isosurface of $\phi_{v}=0.5$ is expressed by four typical vortex lines, and the boundary of LEV and TIV with $\omega_{x}=0$ is highlighted in the blue dashed box. upstream part generated from the leading edge is referred to as the leading edge vortex (LEV). Furthermore, a formal definition of the $\mathrm{LEV}$ is given in Sec. IV.

At the downstream, names of wake vortices are not consistent in the literature, e.g., the tip vortex (TIV) ${ }^{20}$ or the trailing edge vortex (TEV). ${ }^{60}$ In this study, the TIV denotes the streamwise stretching vortex tubes created from the tips; the TEV denotes the vortex tube dominated by the spanwise vorticity, which sheds off from the trailing edge and links two TIVs. Both TIV and TEV are parts of the semivortex ring.

In Figs. 4(c) and 4(e), the LEV keeps lifting and evolves into a hairpinlike structure. The growing curvature of the vortex lines tilting toward the $x$ - and $z$-directions intensifies the self-induced velocity and enhances the elevation of the LEV. The development of the LEV and the suppression of the TIV are described in detail in Sec. IV. Then, the LEV gradually sheds off and propagates downstream, and a secondary hairpinlike structure can form from the bulge for large $R_{A}$ via the similar mechanism in transitional wall flows. ${ }^{4}$

The evolution of the TIV can be simplified by the horseshoe vortex model in the finite wing theory. ${ }^{60}$ In the streamwise cross section, the point vortex model with circulation $\pm \Gamma$ is applied to approximate the vortex pairs in Fig. 8(a). The TIV induces the fluid moving toward the minus $z$-direction, i.e., the downwash. The distance between neighboring VSF isosurfaces linking tip vortex pairs decreases owing to the swirling motion of TIVs, and the size of TIVs grows in Fig. 8(b). At later times, the semiring structure composed of the TIVs and TEV gradually forms and moves downstream, and the portion of the VSF isosurface linking TIVs becomes too thin to be resolved in Fig. 4(e) using a finite grid resolution.

Within the TIV, the evolution of different VSF isosurfaces consisting of helical vortex lines is nearly self-similar and axisymmetric. In Figs. 4(e) and 4(g), the helical degree is intensified with the development of TIV in the wake and the vortex tube is twisted with time. The detailed quantification of the helical TIV is given in Sec. V.

In Appendix B, we investigate the evolution of VSFs in the flows past a plate with various $R e, \alpha$, and $R_{A}$. In general, the vortical structures are similar for larger $R e$ but with faster formation of LEV and TIV. For the LEV formation, the hairpinlike structure does not form with small $\alpha$, and the suppression from the TIV is intensified with smaller $R_{A}$. 

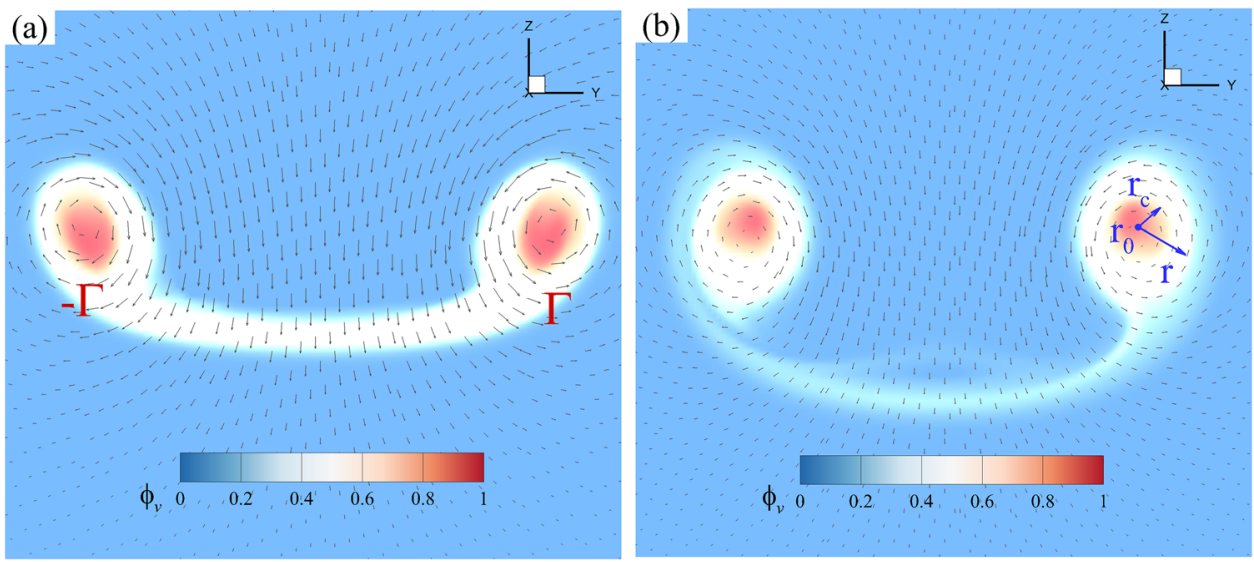

FIG. 8. The VSF contour and velocity vectors on the $y-z$ plane at $x=2.0( \pm \Gamma$ : circulation of TIVs; $r_{0}$ : location of the vortex core; $r_{c}$ : radius of the vortex core radius; $r$ : radial position). (a) $t=2.6$ and (b) $t=4.0$

\section{COMPETITION BETWEEN LEV AND TIV}

\section{A. Streamwise development of the LEV}

The evolution of vortex surfaces in Fig. 4 illustrates that the growing LEV and TIV compete with each other in the tip region, but this three-dimensional feature lacks quantitative analysis and the boundary between the LEV and TIV identified by Eulerian vortex-identification methods is ambiguous. Instead, the VSF can effectively distinguish the LEV and TIV and quantify their interaction. (a)

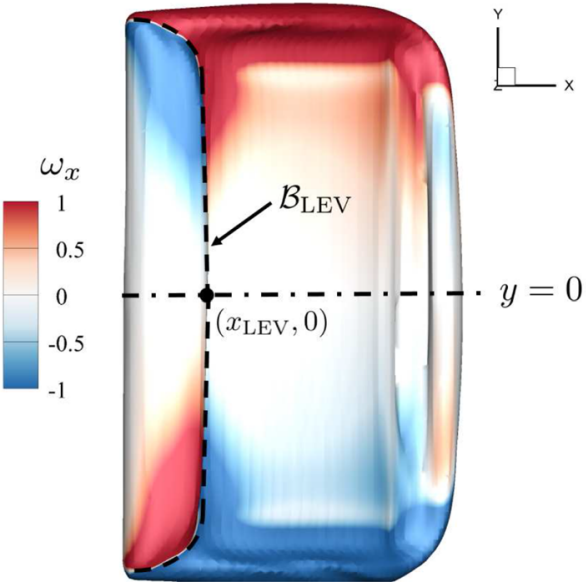

(b)

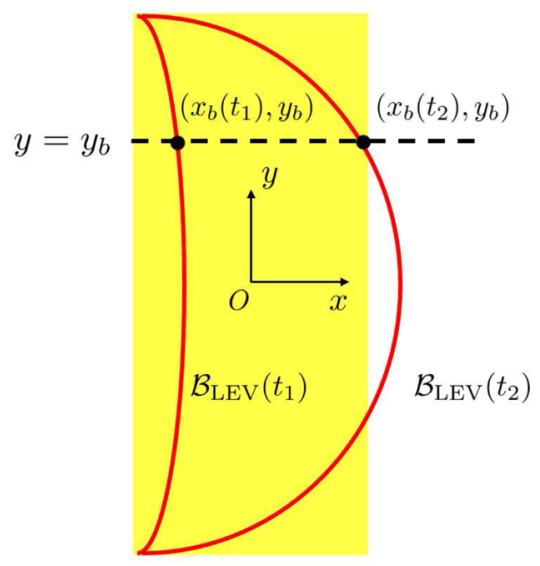

(c)

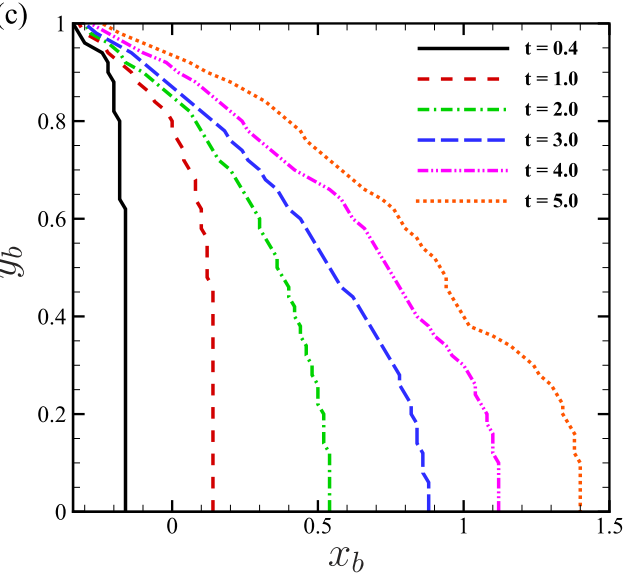

FIG. 9. The evolution of the boundary line $\mathcal{B}_{\text {LEV }}$ of the LEV on the $x$-y plane. (a) The isosurface of $\phi_{v}=0.5$ color-coded by $\omega_{x}$ with $\mathcal{B}_{\mathrm{LEV}}$ at $t=0.4$. (b) The sketch of $\mathcal{B}_{\mathrm{LEV}}$ at different times. (c) The temporal evolution of $\mathcal{B}_{\mathrm{LEV}}$ in terms of $\left(x_{b}\right.$, $\left.y_{b}\right)$. 
As discussed in Sec. III D, the VSF isosurface of $\omega_{x}=0$, as the pair of slanting grooves in Fig. 4, distinguishes LEV and TIV. To characterize the evolution of LEV, we project the boundary $\mathcal{B}_{\text {LEV }}$ between LEV and TIV onto the $x-y$ plane in Fig. 9(a). Considering a half of spanwise region with $0 \leq y_{b} \leq 1$ due to the symmetry, we define

$$
\begin{aligned}
\mathcal{B}_{\mathrm{LEV}}\left(0 \leq y_{b} \leq 1\right)= & \left\{\left(x_{b}, y_{b}\right) \mid x_{b}=\arg \max _{x}\right. \\
& {\left.\left[\phi_{v}\left(x, y_{b}\right) \geq C_{\phi}, \omega_{x}\left(x, y_{b}\right) \leq 0, x \leq x_{\mathrm{LEV}}\right]\right\}, }
\end{aligned}
$$

where $\left(x_{b}, y_{b}\right)$ denotes the coordinate of boundary points, and the LEV location $x_{\mathrm{LEV}}$ is the maximum streamwise coordinate on $\mathcal{B}_{\mathrm{LEV}}$. For example, we mark $\mathcal{B}_{\mathrm{LEV}}$ at $t=0.4$ in Fig. 9(a) and sketch its evolution in Fig. 9(b). It is noted that the VSF threshold is chosen as $C_{\phi}$ $=0.5$ in Eq. (20). This intermediate $C_{\phi}$ for $0 \leq \phi_{v} \leq 1$ can display the vortex surfaces with large deformation ${ }^{44}$ as shown in Fig. 4 and, in particular, can effectively characterize the LEV/TIV competition and the helical TIV in the following discussions.

The evolution of $\mathcal{B}_{\mathrm{LEV}}$ in Fig. 9 (c) quantifies that $x_{b}$ increases with time for all $y_{b}$ and $x_{b}$ decreases with $y_{b}$ owing to the intensified suppression by the TIV. Specifically, the LEV stretches in the streamwise direction and narrows in the spanwise direction, forming a bulgelike structure in Fig. 4(e).

\section{B. Suppression of growing LEV and TIV}

We quantify the suppression of growing LEV and TIV by each other via comparing the nondimensional circulations through cross sections of vortex surfaces. The streamwise circulation $\Gamma_{x}=\int_{A_{y z}} \omega_{x} d A$ and the spanwise circulation $\Gamma_{y}=\int_{A_{x z}} \omega_{y} d A^{6}$ are, respectively, defined on $y-z$ and $x-z$ planes with $\phi_{v}>C_{\phi}$. The spanwise circulation is proportional to the sectional lift force from the Kutta-Joukowski theorem, and Ford and Babinsky ${ }^{61}$ approximated the spanwise lift coefficient by

$$
C_{l}(y)=2 \Gamma_{y}
$$

In Fig. 10(a), the temporal growth of $\Gamma_{y}$ within the LEV is due to the persistent injection of vorticity from the shear layer and contributes to the growth of $C_{l}$ by Eq. (21). In Fig. 10(b), the TIV grows to a matured state with a finite size, as $\Gamma_{x}$ peaks at $t=2 \sim 3$. The growth of $\Gamma_{x}$ from $x=-0.4$ to $x=0.4$ at $t>3.0$ indicates that TIVs evolve into conical structures along the tips in Figs. $4(\mathrm{e})$ and $4(\mathrm{~g})$.

To quantify the competition between LEV and TIV, we define the ratio of spanwise and streamwise circulations through $\mathcal{B}_{\text {LEV }}$ as

$$
R_{\Gamma}\left(\mathcal{B}_{\mathrm{LEV}}\right)=\frac{\Gamma_{\mathrm{LEV}}}{\Gamma_{\mathrm{TIV}}}=\frac{\int_{A_{x z}} \omega_{y}\left(x<x_{b}, y_{b}\right) d A}{\int_{A_{y z}} \omega_{x}\left(x_{b}, y>y_{b}\right) d A} .
$$

(a)

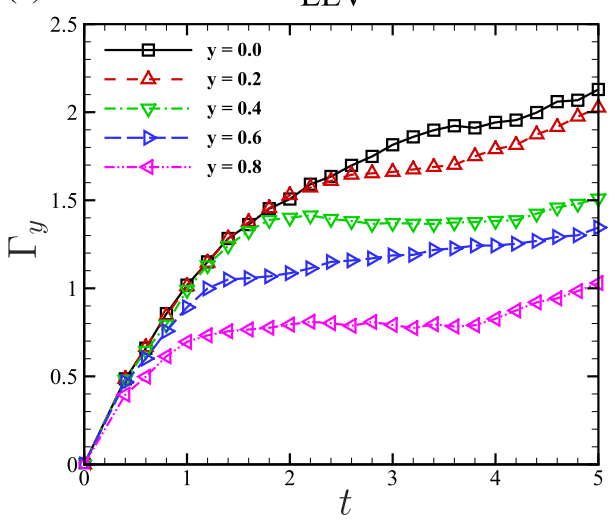

(b)

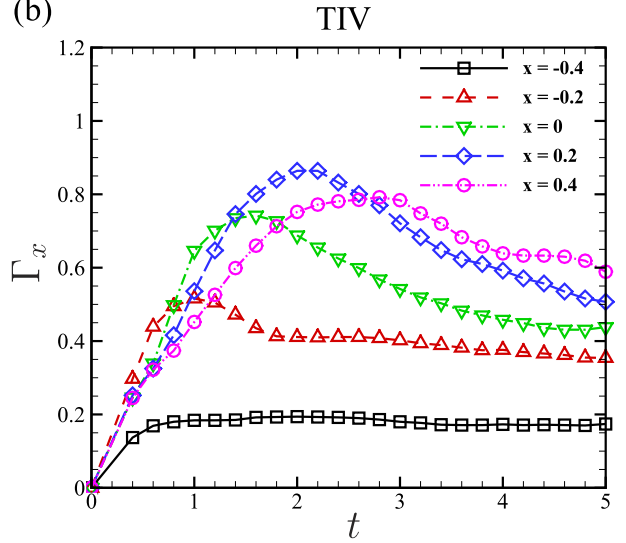

(c)

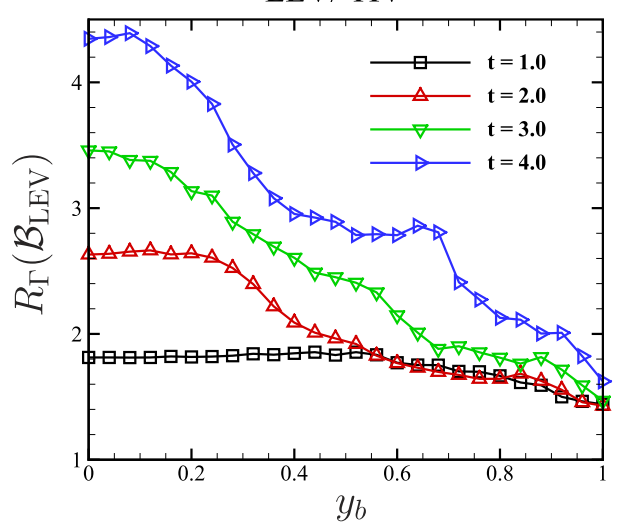

FIG. 10. Characterization of the competition between LEV and TIV. (a) Spanwise circulation for LEV, (b) streamwise circulation for TIV, and (c) the ratio of spanwise and streamwise circulations through $\mathcal{B}_{\mathrm{LEV}}$. 
Figure 10 (c) shows that $R_{\Gamma}\left(\mathcal{B}_{\mathrm{LEV}}\right)$ decreases with $y_{b}$, indicating the suppression of LEV by intensified TIV near the tips. At the early time $t=1.0$, intensities of LEV and TIV are comparable, and they compete with each other around $\mathcal{B}_{\mathrm{LEV}}$. Subsequently, the LEV grows faster than the TIV, as $R_{\Gamma}$ rises with time owing to the finite growth of the TIV. In the late stage, the TIV is not strong enough to suppress the growth of the LEV, as the thinning vortex tubes with diminishing $|\boldsymbol{\omega}|$ in Figs. $4(\mathrm{e})$ and $4(\mathrm{~g})$, and $\mathcal{B}_{\mathrm{LEV}}$ spreads toward the tip region in Fig. 9(c).

\section{FORMATION OF HELICAL TIV}

\section{A. Characterization of the helical TIV}

Since helical vortex lines are attached on the VSF isosurface in Fig. 4, the VSF is useful to characterize the streamwise development of helical TIVs in the wake. Based on the VSF, we define the vortex center at specified $x$ by

$$
\boldsymbol{r}_{0}(x)=\left(y_{c}(x), z_{c}(x)\right), \text { with }\left(y_{c}, z_{c}\right)=\arg \max _{y, z} \phi_{v}(y, z),
$$

where $\boldsymbol{r}_{0}$ is marked in Fig. 8(b) and the radius $r_{c}$ of the vortex core is defined by the distance from $\boldsymbol{r}_{0}$ to the location where the azimuthal velocity on the $y-z$ plane reaches its peak value.

Furthermore, as sketched in Fig. 11, a local cylindrical coordinate system $O-r \theta x$ is employed to analyze the generation of helical vortex lines in TIV tubes. Here, we assume that the central axis of the TIV tube is parallel to the $x$-direction from the observation in Fig. 4.

In order to characterize the helical vortex lines observed in wakes in Figs. 4(e) and 4(g), we define the circumferential average of the helicity density along the radial direction on the $y$ - $z$ plane as

$$
\langle h(r)\rangle_{y z}=\frac{1}{2 \pi} \int_{0}^{2 \pi} \boldsymbol{\omega}(r, \theta) \cdot \boldsymbol{u}(r, \theta) d \theta .
$$

Figure 12 plots the distribution of $\langle h(r)\rangle_{y z}$ against $r$ at three streamwise locations at $t=4.0$. The helicity magnitude diminishes along $x$ owing to the attenuation of the circumferential velocity and vorticity. At each $x,\langle h(r)\rangle_{y z}$ peaks at $r / r_{c} \simeq 0.8$ corresponding to $\phi_{v} \simeq 0.5$. Thus, the VSF threshold $C_{\phi}=0.5$ chosen in this study displays the typical twisting vortex lines on TIV tubes in the wake.

The generation mechanism of the helical TIV is explained as follows: With the rolling up of the TIV in Fig. 4(c), the streamwise velocity gradient $\nabla u_{x}$ along the $r$-direction of TIV tubes generates the circumferential vorticity $\omega_{\theta}$ on the $y-z$ plane. Thus, the stretched vortex lines in the streamwise direction accompanied by the helical development around the vortex axis owing to finite $\omega_{\theta}$ and $\omega_{x}$, and a sequence of helical vortex lines weave into the helical TIV in the wake.

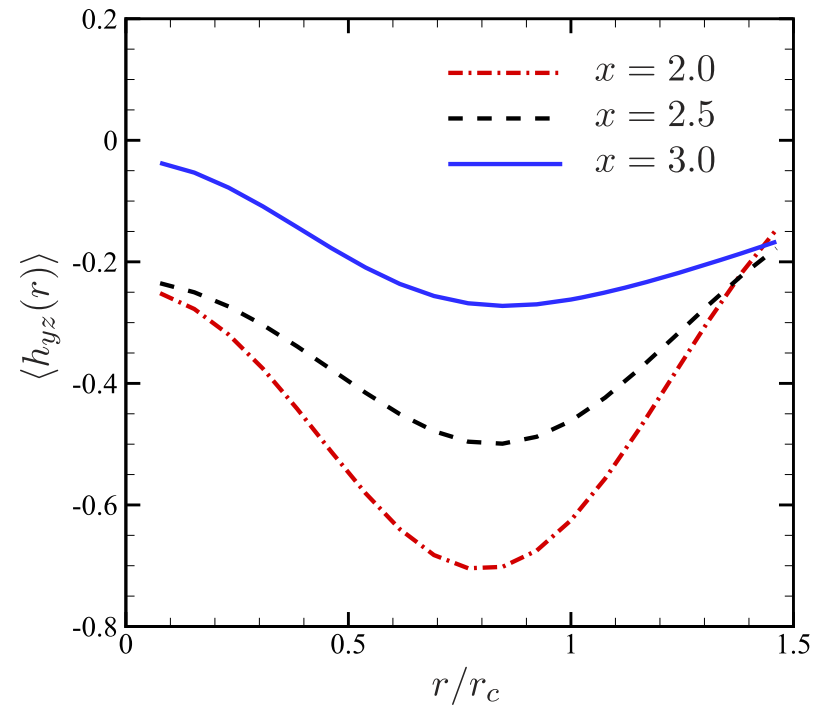

FIG. 12. The circumferential average of the helicity density along the radial direction at four streamwise locations at $t=4.0$.

\section{B. Helical vortex model}

As sketched in Fig. 11, we model the helical TIV tube along the streamwise direction as a straight helical vortex tube. Assuming a helical vortex line with constant pitch $p$ owing to the self-similarity of TIVs in the wake, ${ }^{31}$ the pitch angle $\beta$ quantifies the helical degree of the vortex line $e^{25}$ as

$$
\tan \beta=\frac{\omega_{x}}{\omega_{\theta}}=\frac{p}{2 \pi r},
$$

i.e., the helical degree of vortex lines is inversely proportional to $\beta$. For qualitative analysis, the axial and circumferential vorticities are approximated by Batchelor's vortex $\operatorname{model}^{28,29}$ as

$$
\omega_{\theta} \sim r \exp \left(-B_{1} r^{2}\right) \text { and } \omega_{x} \sim \exp \left(-B_{2} r^{2}\right)
$$

with positive model constants $B_{1}$ and $B_{2}$. As $r \rightarrow 0$, the vortex line becomes rectilinear. For finite $r, \omega_{\theta}$ increases and $\omega_{x}$ decreases with $r$, contributing to the growth of the helical degree by Eq. (25).

Figure 13(a) plots that the helical degree increases with decreasing $\phi_{v}$ or increasing $r$, consistent with the visualization in Fig. 13(b). The nearly collapse of profiles of $\beta$ against $\phi_{v}$ indicates that the helical degree of the TIV is similar from $x=1.5$ to 3.0 at $t=4$. On the other hand, very different profiles of $\beta$ are observed at $x=1$ and $x$ $=3.5$ owing to the influence of LEV and TEV. These two coordinates can be considered as the start and end locations of the helical TIV, so the length of the helical TIV is around 2.0. Considering the
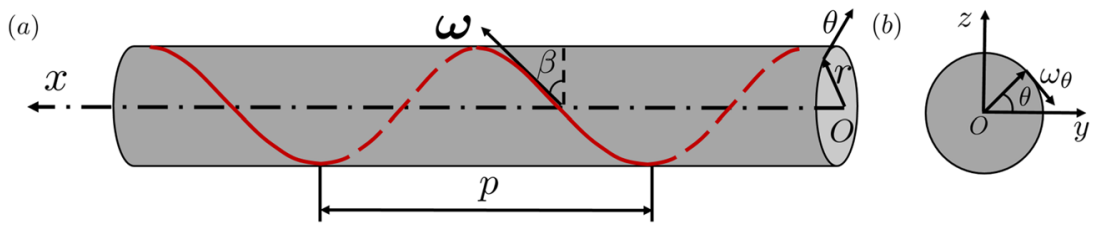

FIG. 11. The local cylindrical coordinate system $0-r \theta x$ in the vortex model for TIV tubes. The red line denotes a vortex line attached on the vortex tube. (a) Perspective view and (b) front view. 

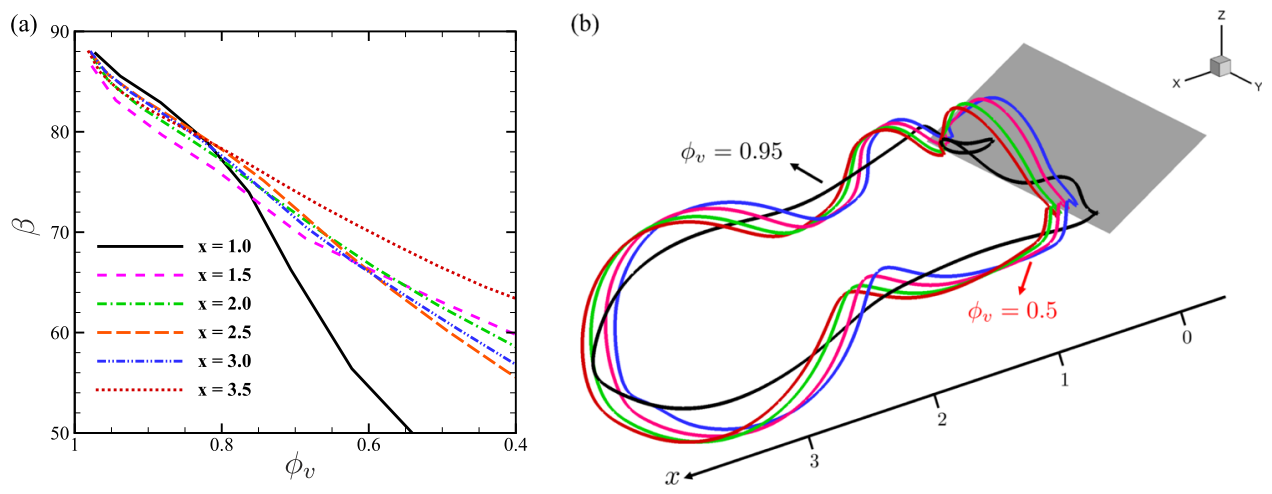

FIG. 13. Characterization of the helical TIV at $t=4$. (a) Profiles of $\beta$ against $\phi_{v}$ at six streamwise locations. (b) Visualization of helical vortex lines on VSF isosurfaces. The black vortex line is integrated on the isosurface of $\phi_{v}=0.95$, and the other vortex lines are integrated on the isosurface of $\phi_{v}=0.5$.

pitch $p=2 \pi r_{\phi} \tan \beta \approx 1.96$ from Eq. (25), where $r_{\phi}$ is the radial distance from $\boldsymbol{r}_{0}$ to the isoline of $\phi_{v}=0.5$ on a $y-z$ plane, the vortex lines in TIV tubes constitute more than one complete helix.

The pitch angle $\beta \simeq 60^{\circ}$ for the isosurface of $\phi_{v}=0.5$ implies a high helical degree of vortex lines, as visualized in Fig. 13(b). The helix of a vortex line is quantified by the rotation angle

$$
\gamma(t) \sim 2 \pi \frac{l(t)}{p}=\frac{l(t)}{r_{\phi} \tan \beta}
$$

around the vortex axis, where the length $l(t)$ of the wake grows with time. Hence, stronger helical degree of vortex lines with increasing $\gamma$ is observed with the streamwise development of the TIV in Fig. 4.

\section{Effects of helical vortex lines on drag and lift forces}

The downwash induced by TIVs has an impact on drag and lift forces on the plate. Based on the VSF and the impulse theory, we quantify the vortical impulse generated by the TIV enclosed by a particular vortex surface. The streamwise and vertical vortical

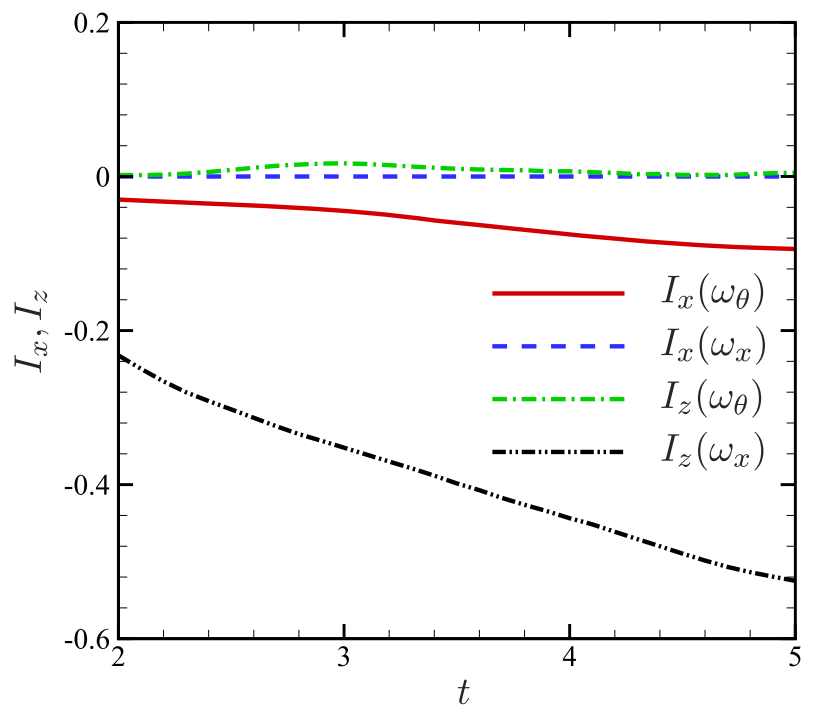

FIG. 14. Decomposition of vortical impulses of the TIV. impulses of the TIV are defined by

$$
I_{x}=\frac{1}{2} \int_{\mathcal{V}}\left(y \omega_{z}-z \omega_{y}\right) \mathrm{d} V \text { and } I_{z}=\frac{1}{2} \int_{\mathcal{V}}\left(x \omega_{y}-y \omega_{x}\right) \mathrm{d} V,
$$

where $\mathcal{V}$ is the total volume of the TIV with $\phi_{v}>C_{\phi}$.

To quantify effects of the helical vortex lines on the vortical impulse, we decompose the vorticity into the helical component $\omega_{\theta}$ and the axial component $\omega_{x}$. Based on the vortex model in Fig. 11(b), substituting

$$
\omega_{y}=\omega_{\theta} \sin \theta \text { and } \omega_{z}=-\omega_{\theta} \cos \theta
$$

on the $y-z$ plane into Eq. (28) yields the decomposition of $I_{x}$ and $I_{z}$ in terms of $\omega_{\theta}$ and $\omega_{x}$ as

$$
\begin{aligned}
& I_{x}\left(\omega_{\theta}\right)=-\int_{\mathcal{V}} \pi \omega_{\theta} r \mathrm{~d} x \mathrm{~d} r \text { and } I_{x}\left(\omega_{x}\right)=0, \\
& I_{z}\left(\omega_{\theta}\right)=\frac{1}{2} \int_{\mathcal{V}} x \omega_{y} \mathrm{~d} V=0 \text { and } I_{z}\left(\omega_{x}\right)=\frac{1}{2} \int_{\mathcal{V}}-y \omega_{x} \mathrm{~d} V .
\end{aligned}
$$

We calculate $I_{x}$ and $I_{z}$ by Eqs. (28) and (29), and the dependence of $I_{x}$ and $I_{z}$ on $\omega_{\theta}$ and $\omega_{x}$ is consistent with the approximations [Eqs. (30) and (31)] in Fig. 14. If we assume that the helical TIVs are isolated from other vortical structures, drag and lift forces are time derivatives of $I_{x}$ and $I_{z}$, respectively, in the impulse theory. Thus, $I_{z}$ and the lift are dominated by the time history of $\omega_{x}$, and $I_{x}$ and the drag are determined by the time history of $\omega_{\theta}$, which implies that we can reduce the drag by suppressing the generation of $\omega_{\theta}$. On the other hand, the interaction of LEV and TIV can be complex, and the effect of helical TIV on the forces should be scrutinized in aerodynamic applications.

\section{CONCLUSIONS}

We investigate the evolution of the VSF in the threedimensional flow past a finite plate at $R e=300, R_{A}=2$, and $\alpha=30^{\circ}$. The immersed boundary method is applied to obtain the velocityvorticity field, and the two-time VSF method is extended to the immersed boundary by adding a source term in the VSF evolution equation. The numerical VSF solution with negligible VSF deviation is effective to characterize the three-dimensional features of vortex surfaces. 
The VSF evolution can be roughly divided into three stages based on the morphology of vortex surfaces and lines. At the early time, vortex surfaces keep flat and ringlike vortex lines are stretched in the streamwise direction. Then, near-plate vortex surfaces roll up from plate edges and form a dustpanlike structure. In the late stage, a hairpinlike structure is generated near the leading edge and a semiring structure is formed near plate tips and in the wake. We quantitatively distinguish the LEV and TIV by the vanishing streamwise vorticity on the VSF isosurfaces, and the formally defined interface is used for further quantitative analysis.

The competition between the LEV and TIV is characterized based on the VSF. We quantify the streamwise development of the LEV by tracking the vanishing streamwise vorticity on VSF isosurfaces and find that the LEV is suppressed near the tip region. The intensities of LEV and TIV are defined by the corresponding circulations through cross sections enclosed by the VSF isosurface. Then, we use the ratio of circulations of LEV and TIV to elucidate that the development of the LEV near the tips is suppressed by the finite growth of the TIV and the suppression is mitigated near the wake.

In the wake region, helical vortex lines are generated in TIV tubes, and they are characterized by self-similar profiles of the pitch angle of vortex lines on the VSF isosurface. We demonstrate that the helical vorticity component dominates the streamwise vortical impulse, so the suppression of helical degree of TIV tubes can facilitate the drag reduction.

In the future work, the VSF can be extended to analyze the forces in various moving-boundary problems, particularly in biofluid dynamics, which helps us to improve flow control techniques through quantitative relations between forces and vortical structures. For the minimum-domain impulse theory, ${ }^{35}$ a special surface domain without cutting vorticity can be chosen to eliminate surface integrals in the vortex-force formula. Thus, the integral domain enclosed by the vortex surface is useful to simplify the calculation of vortex forces owing to the vanishing normal vorticity on the domain boundary. Additionally, the VSF can be applied to temporally resolved PIV data for elucidating continuous vortex dynamics through the experiment of flows past a plate.

\section{ACKNOWLEDGMENTS}

Numerical simulations were carried out on the TH-2A supercomputer in Guangzhou, China. This work was supported in part by the National Natural Science Foundation of China (Grant Nos. 91952108, 11925201, and 91841302).

\section{APPENDIX A: CALCULATION OF THE VSF EVOLUTION EQUATION WITH A SOURCE TERM}

Similar to the direct forcing scheme for the immersed boundary method, we apply the direct source method to solve Eq. (9). Within the substep for advancing the time step from $n$ to $n+1$, the temporary VSF solution is obtained by the procedure as follows:

First, Eq. (9) is solved without the source term $q$ as

$$
\frac{\tilde{\phi}_{v}^{*}-\phi_{v}^{* n}}{\Delta t}+\boldsymbol{u}(\boldsymbol{x}, t) \cdot \nabla \phi_{v}^{* n}(\boldsymbol{x}, t)=0 .
$$

Then, we calculate the VSF $\tilde{\phi}_{v}^{B}$ predicted at the boundary using the interpolation equation (11) as

$$
\tilde{\phi}_{v}^{B}(\boldsymbol{X})=\sum_{x} \tilde{\phi}_{v}^{*}(\boldsymbol{x}) \delta_{h}(\boldsymbol{x}-\boldsymbol{X})(\Delta x)^{3}
$$

Here, the regularized discrete delta function ${ }^{50}$ is

$$
\delta_{h}(\boldsymbol{x}-\boldsymbol{X})=\frac{1}{(\Delta x)^{3}} \phi\left(\frac{x-X}{\Delta x}\right) \phi\left(\frac{y-Y}{\Delta x}\right) \phi\left(\frac{z-Z}{\Delta x}\right),
$$

with $\boldsymbol{x}=(x, y, z), \boldsymbol{X}=(X, Y, Z)$, and

$$
\phi(r)= \begin{cases}\frac{1}{8}\left(3-2|r|+\sqrt{1+4|r|-4 r^{2}}\right), & |r| \leqslant 1, \\ \frac{1}{8}\left(5-2|r|-\sqrt{-7+12|r|-4 r^{2}}\right), & 1<|r|<2, \\ 0, \quad 2 \leqslant|r| . & \end{cases}
$$

Subsequently the Lagrangian source term $Q(X)$ is applied to enforce the immersed boundary condition by

$$
Q(X)=\frac{\phi_{v}^{B}(\boldsymbol{X})-\tilde{\phi}_{v}^{B}(\boldsymbol{X})}{\Delta t},
$$

and it is spread to the flow field using a discrete form of Eq. (12) as

$$
q(\boldsymbol{x})=\sum_{k=1}^{M} Q\left(\boldsymbol{X}_{k}\right) \delta_{h}\left(\boldsymbol{x}-\boldsymbol{X}_{k}\right)(\Delta s)^{3},
$$

where $M$ is the total number of Lagrangian points and $\Delta s$ is the distance of two neighboring Lagrangian points approximately equivalent to $\Delta x$. Finally, the temporary VSF is advanced as

$$
\phi_{v}^{* n+1}=\tilde{\phi}_{v}^{*}+q \Delta t .
$$

\section{APPENDIX B: VSF EVOLUTION IN FLOWS PAST A PLATE WITH VARIOUS PARAMETERS}

We investigate the effects of $R e, \alpha$, and $R_{A}$ on the VSF evolution in flows past a finite plate to reveal the relationship between the lift coefficient $C_{L}$ and vortical structures. Here, the lift coefficient is calculated by

$$
C_{L}=\frac{\sum_{k=1}^{M} F_{z}\left(X_{k}\right)(\Delta s)^{3}}{\frac{1}{2} \rho U_{\infty}^{2} R_{A}}
$$

where $F_{z}$ denotes the component of the Lagrangian force in the $z$ direction.

First, the shedding of vortical structures occurs earlier for higher $R e .^{19}$ Figure 15(a) shows the isosurface of VSF at $t=5$ for $R e=500$. The primary hairpinlike vortex sheds earlier, and the TIV shows stronger distortion compared with that in Fig. 4(g). The faster formation of the LEV contributes to larger $C_{L}$ in Fig. 16(a). Second, the vortex shedding does not occur at small $\alpha$. The VSF isosurface at $\alpha=10^{\circ}$ in Fig. 15(b) depicts the almost flat LEV with the small spanwise circulation and thin TIV tubes with weakly helical vortex lines. With $\alpha$ decreasing from $30^{\circ}$ to $10^{\circ}$, the rolling up of vortical structures on plate edges is significantly suppressed. Thus, $C_{L}$ is reduced with decreasing $\alpha$ in Fig. 16(a). Finally, the suppression of TIVs on the lift of LEV is intensified with smaller $R_{A}$ from the comparison between Figs. 15(c) and 4(g). As discussed in Sec. IV B, the spanwise circulation in the LEV is a major source of the lift force, so $C_{L}$ with $R_{A}=1$ is smaller than that with $R_{A}=2$ in Fig. 16(a). 
(a)

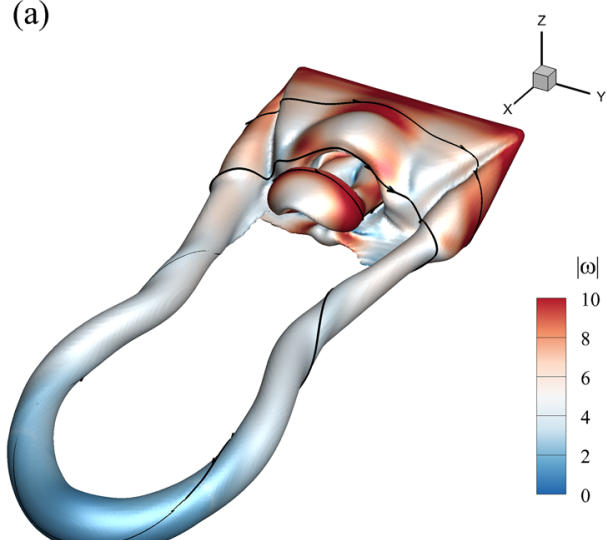

(b)

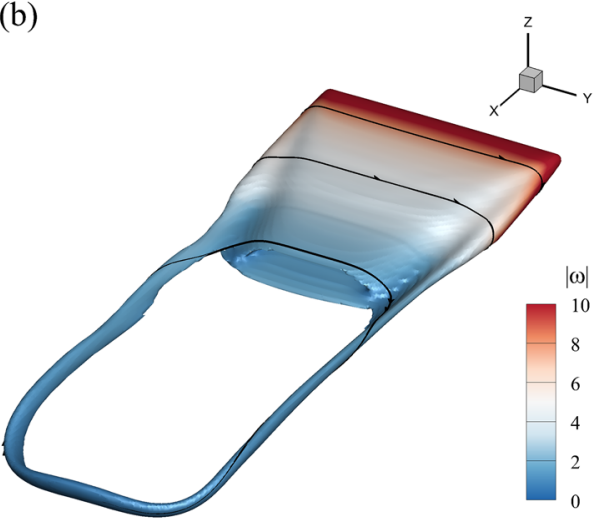

(c)

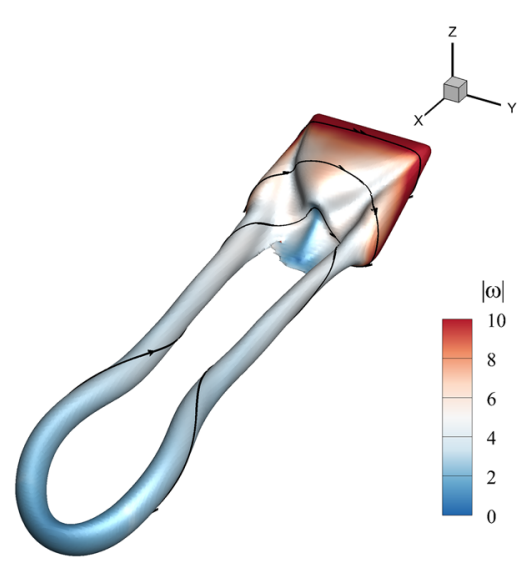

FIG. 15. Comparison of VSF isosurfaces with various $R e, \alpha$, and $R_{A}$. (a) $R e=500$, $\alpha=30^{\circ}$, and $R_{A}=2$ at $t=5 ;$ (b) $R e=300$, $\alpha=10^{\circ}$, and $R_{A}=2$ at $t=5$; and (c) $R e$ $=300, \alpha=30^{\circ}$, and $R_{A}=1$ at $t=5$.
To quantify the LEV/TIV competition with various $R e, \alpha$, and $R_{A}$, we compare the ratio of spanwise and streamwise circulations through $\mathcal{B}_{\text {LEV }}$ at $t=4$ in Fig. $16(\mathrm{~b})$, where $y_{b}^{*}=2 y_{b} / R_{A}$ denotes the normalized spanwise location. The profiles of $R_{\Gamma}\left(\mathcal{B}_{\mathrm{LEV}}\right)$ against $y_{b}^{*}$ indicate the similar competition between a bulgelike LEV and TIVs at different $R e$ and $R_{A}$. By contrast, the TIV is very weak and the LEV remains almost flat for $\alpha=10^{\circ}$ in Fig. 15(b). Thus, $R_{\Gamma}\left(\mathcal{B}_{\mathrm{LEV}}\right)$ for this small $\alpha$ case is distinguished from those in other three cases in Fig. 16(b).

In the wake, we observe helical vortex lines of TIVs in all the cases, and the helical degree varies with $R e, \alpha$, and $R_{A}$. From the calculation of pitch angles at $t=4$ from Eq. (25), we observe a higher helical degree of vortex lines with smaller $\beta=54^{\circ}$ for larger $R e$ $=500$, also shown as the stronger distortion of the VSF isosurface (a)

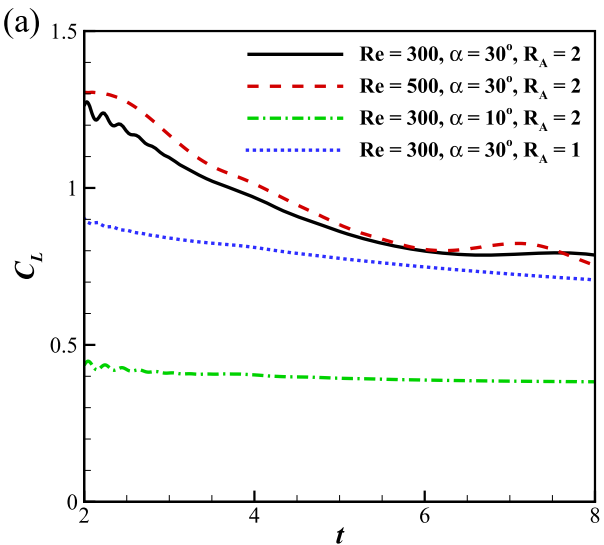

(b)

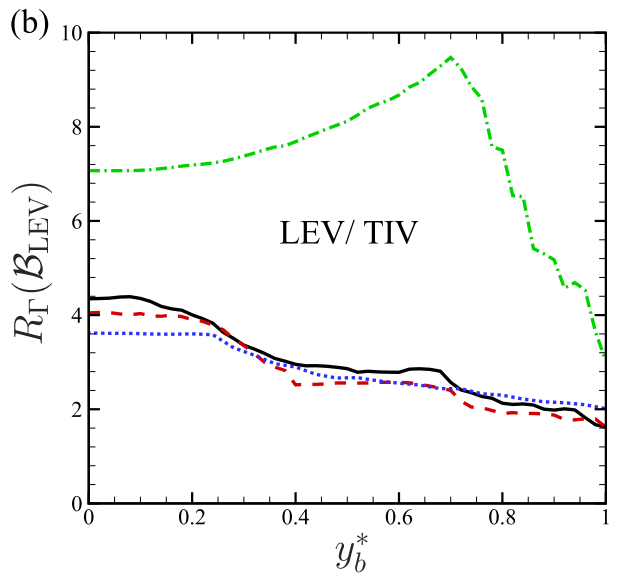

FIG. 16. Effects of $R e, \alpha$, and $R_{A}$ on the lift coefficient and LEV/TIV competition. (a) Temporal evolution of the lift coefficient and (b) the ratio of spanwise and streamwise circulations through $\mathcal{B}_{\mathrm{LEV}}$ at $t=4$. 
in Fig. 15(a). For smaller $R_{A}=1$, the TIV is slender and $\beta$ increases to $70^{\circ}$. For smaller $\alpha=10^{\circ}$, the TIV shows the weakest helical degree with $\beta=75^{\circ}$.

\section{REFERENCES}

${ }^{1}$ W. Shyy, H. Aono, S. K. Chimakurthi, P. Trizila, C.-K. Kang, C. E. S. Cesnik, and H. Liu, "Recent progress in flapping wing aerodynamics and aeroelasticity," Prog. Aerosp. Sci. 46, 284-327 (2010).

${ }^{2}$ C. P. Ellington, C. Van Den Berg, A. P. Willmott, and A. L. R. Thomas, "Leadingedge vortices in insect flight," Nature 384, 626-630 (1996).

${ }^{3}$ J. M. Birch and M. H. Dickinson, "Spanwise flow and the attachment of the leading-edge vortex on insect wings," Nature 412, 729-733 (2001).

${ }^{4}$ Y. Z. Lyu, H. J. Zhu, and M. Sun, "Aerodynamic forces and vortical structures of a flapping wing at very low Reynolds numbers," Phys. Fluids 31, 041901 (2019).

${ }^{5}$ A.-J. Buchner, N. Buchmann, K. Kilany, C. Atkinson, and J. Soria, "Stereoscopic and tomographic PIV of a pitching plate," Exp. Fluids 52, 299-314 (2012).

${ }^{6} \mathrm{~T}$. Jardin, A. Farcy, and L. David, "Three-dimensional effects in hovering flapping flight," J. Fluid Mech. 702, 102-125 (2012).

${ }^{7} \mathrm{O}$. Son and O. Cetiner, "Three-dimensionality effects due to change in the aspect ratio for the flow around an impulsively pitching flat plate," J. Aerosp. Eng. 30, 04017053 (2017).

${ }^{8}$ J. T. King, R. Kumar, and M. A. Green, "Experimental observations of the three-dimensional wake structures and dynamics generated by a rigid, bioinspired pitching panel," Phys. Rev. Fluids 3, 034701 (2018).

${ }^{9}$ S.-F. Zou, J. Z. Wu, A.-K. Gao, L. Liu, L. Kang, and Y. Shi, "On the concept and theory of induced drag for viscous and incompressible steady flow," Phys. Fluids 31, 065106 (2019).

${ }^{10}$ J. H. J. Buchholz and A. J. Smits, "On the evolution of the wake structure produced by a low-aspect-ratio pitching panel," J. Fluid Mech. 546, 433-443 (2006).

${ }^{11}$ J. H. J. Buchholz and A. J. Smits, "The wake structure and thrust performance of a rigid low-aspect-ratio pitching panel," J. Fluid Mech. 603, 331-365 (2008).

${ }^{12}$ M. Rahromostaqim, A. Posa, and E. Balaras, "Numerical investigation of the performance of pitching airfoils at high amplitudes," AIAA J. 54, 2221-2232 (2016).

${ }^{13}$ P. Mancini, F. Manar, K. Granlund, M. V. Ol, and A. R. Jones, "Unsteady aerodynamic characteristics of a translating rigid wing at low Reynolds number," Phys. Fluids 27, 123102 (2015).

${ }^{14} \mathrm{~S}$. Wang, G. He, and X. Zhang, "Lift enhancement on spanwise oscillating flatplates in low-Reynolds-number flows," Phys. Fluids 27, 061901 (2015).

${ }^{15} \mathrm{~J}$. N. Fernando and D. E. Rival, "On vortex evolution in the wake of axisymmetric and non-axisymmetric low-aspect-ratio accelerating plates," Phys. Fluids 28, 017102 (2016).

${ }^{16} \mathrm{D}$. Kim and M. Gharib, "Experimental study of three-dimensional vortex structures in translating and rotating plates," Exp. Fluids 49, 329-339 (2010).

${ }^{17}$ T. O. Yilmaz and D. Rockwell, "Flow structure on finite-span wings due to pitchup motion,” J. Fluid Mech. 691, 518-545 (2012).

${ }^{18} \mathrm{C}$. Hartloper, M. Kinzel, and D. E. Rival, "On the competition between leadingedge and tip-vortex growth for a pitching plate," Exp. Fluids 54, 1447 (2013).

${ }^{19} \mathrm{~K}$. Taira and T. Colonius, "Three-dimensional flows around low-aspect-ratio flat-plate wings at low Reynolds numbers," J. Fluid Mech. 623, 187-207 (2009).

${ }^{20}$ J.-J. Lee, C.-T. Hsieh, C. C. Chang, and C.-C. Chu, "Vorticity forces on an impulsively started finite plate," J. Fluid Mech. 694, 464-492 (2012).

${ }^{21} \mathrm{C}$. Li and H. Dong, "Three-dimensional wake topology and propulsive performance of low-aspect-ratio pitching-rolling plates," Phys. Fluids 28, 071901 (2016).

${ }^{22}$ G.-J. Li and X.-Y. Lu, "Force and power of flapping plates in a fluid," J. Fluid Mech. 712, 598-613 (2012).

${ }^{23} \mathrm{M}$. R. Visbal, "Unsteady flow structure and loading of a pitching low-aspectratio wing,” Phys. Rev. Fluids 2, 024703 (2017).

${ }^{24} \mathrm{~J}$. D. Eldredge and A. R. Jones, "Leading-edge vortices: Mechanics and modeling," Annu. Rev. Fluid Mech. 51, 75-104 (2019).
${ }^{25}$ R. L. Ricca, "The effect of torsion on the motion of a helical vortex filament," J. Fluid Mech. 273, 241-259 (1994).

${ }^{26}$ S. V. Alekseenko, P. A. Kuibin, V. L. Okulov, and S. I. Shtork, "Helical vortices in swirl flow," J. Fluid Mech. 382, 195-243 (1999).

${ }^{27}$ H. Lamb, Hydrodynamics (Cambridge University Press, 1993).

${ }^{28}$ G. K. Batchelor, "Axial flow in trailing line vortices," J. Fluid Mech. 20, 645-658 (1964).

${ }^{29}$ W. J. Devenport, M. C. Rife, S. I. Liapis, and G. J. Follin, "The structure and development of a wing-tip vortex," J. Fluid Mech. 312, 67-106 (1996).

${ }^{30}$ P. R. Spalart, "Airplane trailing vortices," Annu. Rev. Fluid Mech. 30, 107-138 (1998).

${ }^{31}$ V. L. Okulov, I. K. Kabardin, R. F. Mikkelsen, I. V. Naumov, and J. N. Sørensen, "Helical self-similarity of tip vortex cores," J. Fluid Mech. 859, 1084-1097 (2019).

${ }^{32} \mathrm{~J}$. Wu, L. Liu, and T. Liu, "Fundamental theories of aerodynamic force in viscous and compressible complex flows," Prog. Aerosp. Sci. 99, 27-63 (2018).

${ }^{33}$ S. Wang, G. He, and T. Liu, "Estimating lift from wake velocity data in flapping flight," J. Fluid Mech. 868, 501-537 (2019).

${ }^{34} \mathrm{~J}$. C. Wu, "Theory for aerodynamic force and moment in viscous flows," AIAA J. 19, 432-441 (1981).

${ }^{35}$ L. L. Kang, L. Q. Liu, W. D. Su, and J. Z. Wu, "Minimum-domain impulse theory for unsteady aerodynamic force," Phys. Fluids 30, 016107 (2018).

${ }^{36}$ D. B. Quinn, G. V. Lauder, and A. J. Smits, "Scaling the propulsive performance of heaving flexible panels," J. Fluid Mech. 738, 250-267 (2014).

${ }^{37}$ F. Ayancik, Q. Zhong, D. B. Quinn, A. Brandes, H. Bart-Smith, and K. W. Moored, "Scaling laws for the propulsive performance of three-dimensional pitching propulsors," J. Fluid Mech. 871, 1117-1138 (2019).

${ }^{38}$ B. Epps, "Review of vortex identification methods," in 55th AIAA Aerospace Sciences Meeting (AIAA, 2017), p. 0989.

${ }^{39}$ J.-Z. Wu, "Vortex definition and "vortex criteria"," Sci. China: Phys., Mech. Astron. 61, 024731 (2018).

${ }^{40}$ J. C. R. Hunt, A. A. Wray, and P. Moin, "Eddies, streams, and convergence zones in turbulent flows," in Center for Turbulent Research Report (Stanford University, 1988), Vol. 1, pp. 193-208.

${ }^{41}$ M. S. Chong, A. E. Perry, and B. J. Cantwell, "A general classification of threedimensional flow fields," Phys. Fluids A 2, 765-777 (1990).

${ }^{42}$ J. Jeong and F. Hussain, "On the identification of a vortex," J. Fluid Mech. 285, 69-94 (1995).

${ }^{43}$ Y. Yang and D. I. Pullin, "On Lagrangian and vortex-surface fields for flows with Taylor-Green and Kida-Pelz initial conditions," J. Fluid Mech. 661, 446-481 (2010).

${ }^{44}$ Y. Yang and D. I. Pullin, "Evolution of vortex-surface fields in viscous TaylorGreen and Kida-Pelz flows," J. Fluid Mech. 685, 146-164 (2011).

${ }^{45}$ Y. Zhao, Y. Yang, and S. Chen, "Evolution of material surfaces in the temporal transition in channel flow," J. Fluid Mech. 793, 840-876 (2016).

${ }^{46} \mathrm{Y}$. Zhao, Y. Yang, and S. Chen, "Vortex reconnection in the late transition in channel flow,” J. Fluid Mech. 802(R4), 1-13 (2016).

${ }^{47}$ Y. Zhao, S. Xiong, Y. Yang, and S. Chen, "Sinuous distortion of vortex surfaces in the lateral growth of turbulent spots," Phys. Rev. Fluids 3, 074701 (2018).

${ }^{48} \mathrm{Y}$. Huang and M. A. Green, "Detection and tracking of vortex phenomena using Lagrangian coherent structures," Exp. Fluids 56, 147 (2015).

${ }^{49}$ S. Krishna, M. A. Green, and K. Mulleners, "Flowfield and force evolution for a symmetric hovering flat-plate wing," AIAA J. 56, 1360-1371 (2018).

${ }^{50}$ C. S. Peskin, "The immersed boundary method," Acta Numer. 11, 479-517 (2002).

${ }^{51}$ S. Wang and X. Zhang, "An immersed boundary method based on discrete stream function formulation for two- and three-dimensional incompressible flows," J. Comput. Phys. 230, 3479-3499 (2011).

${ }^{52}$ S. Wang, M. Vanella, and E. Balaras, "A hydrodynamic stress model for simulating turbulence/particle interactions with immersed boundary methods," J. Comput. Phys. 382, 240-263 (2019).

${ }^{53}$ S. Wang, X. Zhang, G. He, and T. Liu, "A lift formula applied to low-Reynoldsnumber unsteady flows," Phys. Fluids 25, 093605 (2013).

${ }^{54}$ R. Franke, "Scattered data interpolation: Tests of some methods," Math. Comput. 38, 181-200 (1982). 
${ }^{55} \mathrm{~S}$. Xiong and Y. Yang, "The boundary-constraint method for constructing vortex-surface fields," J. Comput. Phys. 339, 31-45 (2017).

${ }^{56} \mathrm{~N}$. Zhang, Z. C. Zheng, and S. Eckels, "Study of heat-transfer on the surface of a circular cylinder in flow using an immersed-boundary method," Int. J. Heat Fluid Flow 29, 1558-1566 (2008).

${ }^{57}$ A. Mark, E. Svenning, and F. Edelvik, "An immersed boundary method for simulation of flow with heat transfer," Int. J. Heat Mass Transfer 56, 424-435 (2013).
${ }^{58}$ J.-Z. Wu, H.-Y. Ma, and M.-D. Zhou, Vortical Flows (Springer, 2015).

${ }^{59}$ H. Kaden, "Aufwicklung einer unstabilen unstetigkeitsfläche," Ing.-Arch. 2, $140-168$ (1931).

${ }^{60}$ E. L. Houghton and P. W. Carpenter, Aerodynamics for Engineering Students (Elsevier, 2003).

${ }^{61}$ C. W. P. Ford and H. Babinsky, "Lift and the leading-edge vortex," J. Fluid Mech. 720, 280-313 (2013). 